Cahiers
de la Recherche
surles Droits Fondamentaux
Cahiers de la recherche sur les droits fondamentaux

4 | 2005

Quel avenir pour la laïcité cent ans après la loi de 1905 ?

\title{
La liberté religieuse dans les lieux publics
}

\section{Stéphane Guérard}

\section{(2) OpenEdition}

Journals

Édition électronique

URL : https://journals.openedition.org/crdf/7273

DOI : $10.4000 /$ crdf.7273

ISSN : 2264-1246

Éditeur

Presses universitaires de Caen

Édition imprimée

Date de publication : 1 décembre 2005

Pagination : 49-70

ISBN : 2-84133-250-0

ISSN : $1634-8842$

Référence électronique

Stéphane Guérard, "La liberté religieuse dans les lieux publics », Cahiers de la recherche sur les droits fondamentaux [En ligne], 4 | 2005, mis en ligne le 15 décembre 2020, consulté le 14 novembre 2022. URL : http://journals.openedition.org/crdf/7273 ; DOI : https://doi.org/10.4000/crdf.7273 


\section{La liberté religieuse dans les lieux publics}

\section{Stéphane GUÉRARD}

Maître de conférences à I'Université du droit et de la santé, Lille II

(CERAPS - CNRS - UMR n 8026)

Introduction

A. Les notions de " liberté religieuse " et de " neutralité religieuse "

1. La double "liberté religieuse "

2. La neutralité religieuse

a. La définition de la neutralité religieuse

b. L'impact de la neutralité religieuse sur les lieux publics

B. La notion de "lieu public "

1. Le double sens du " lieu public "

2. Le «lieu public " et la distinction domaniale

3. Le « lieu public " et l'exécution du service public

C. L'histoire de la neutralité religieuse des lieux publics

1. La période précédant le vote de la loi du 9 décembre 1905

2. La loi du 9 décembre 1905

D. Les rencontres des deux pouvoirs dans les lieux publics

E. L'annonce de plan

I. La neutralité religieuse dans les lieux publics : une obligation affirmée

A. Un principe général : I'obligation de neutralité religieuse

1. Une justification essentielle : la non-discrimination religieuse

2. Un exemple classique : les manifestations religieuses sur la voie publique

3. Une limite importante : la liberté religieuse des usagers

B. Une condition importante : le devoir de neutralité des agents publics

1. Le contenu du devoir de neutralité

2. L'intérêt de la neutralité de l'agent public pour celle du lieu public

II. La neutralité religieuse dans les lieux publics : une application nuancée

A. Un principe ébranlé : le lieu funéraire

1. La neutralité religieuse des cimetières

2. La survivance voire la renaissance des cimetières et carrés confessionnels a. Les cimetières confessionnels

b. Les carrés confessionnels

B. Un principe contesté : les lieux publics cultuels

1. Les aumôneries

2. Les édifices cultuels

Conclusion 


\section{Introduction}

La liberté est un bien si précieux, disait Montesquieu, que chacun veut prendre celle des autres ${ }^{1}$.

\section{A. Les notions de "liberté religieuse " et de "neutralité religieuse "}

"C'est la neutralité de l'espace public qui permet la coexistence harmonieuse des différentes religions $»^{2}$.

Mais avant de cerner la notion de «neutralité religieuse » (2), il convient de définir la liberté religieuse (1).

\section{La double « liberté religieuse »}

«La liberté religieuse revêt deux aspects distincts mais complémentaires. Elle signifie tout d'abord le droit de choisir et d'exprimer sa foi, droit qu'on dénomme habituellement la "liberté de conscience" » ${ }^{3}$.

Ensuite, cette liberté recouvre aussi, selon l'article 9 de la Convention européenne de sauvegarde des droits de l'homme et des libertés fondamentales du 4 novembre 1950, le « [...] droit à la liberté de pensée, de conscience et de religion; ce droit implique [...] la liberté de manifester sa religion ou sa conviction individuellement ou collectivement, en public ou en privé, par le culte, l'enseignement, les pratiques et l'accomplissement des rites ». Ce second aspect de la liberté religieuse est appelé «la liberté des cultes $»^{4}$.

Mais, si croire est une action passive, en général discrète, parce que normalement intériorisée, exprimer sa croyance est, quant à elle, une attitude active, extériorisée et qui, de ce fait, peut gêner ceux qui ne croient pas ou croient différemment que le croyant qui exprime sa foi.

Il importe dès lors de s'interroger sur les contraintes juridiques réelles inhérentes à une telle expression en fonction des lieux choisis pour celle-ci.

Ceci est particulièrement problématique au regard de la situation juridique des lieux publics. En effet, et malgré la neutralité principielle affichée de tels lieux, il peut sembler parfois difficile de la respecter strictement au regard de certains de ces lieux, tels que les cimetières, les lieux cultuels voire les voies publiques, par exemple.

Par suite, «Le fait religieux ne peut pas être ignoré par les pouvoirs publics $»^{5}$.

\section{La neutralité religieuse}

\section{a. La définition de la neutralité religieuse}

Tout d'abord, «Qu'est-ce que la neutralité ? [...] Question redoutable $[\ldots] »^{6}$.

Pris dans son acception courante, rester neutre, c'est ne pas prendre parti, ne pas s'engager d'un côté ou de l'autre $^{7}$; ce qui va au-delà de l'égalité.

Toutefois, et dans la mesure où il implique la nondiscrimination à raison de la race, des convictions idéologiques, politiques ou religieuses, le principe de neutralité rejoint le principe d'égalité avec lequel il se confond. La loi nº 2004-1486 du 30 décembre 2004 a d'ailleurs créé une Haute autorité de lutte contre les discriminations et pour l'égalité.

«La neutralité concerne la politique, la philosophie, la religion. Elle englobe donc la laïcité qui ne concerne que cette dernière $»^{8}$.

Neutralité religieuse (donc laïcité) et égalité, ces deux principes fondateurs de la République, vont de pair $^{9}$, ne serait-ce que parce qu'ils sont inscrits dans le texte constitutionnel français du 4 octobre 1958 : ils sont d'ailleurs aujourd'hui proclamés dans le même article, l'article $1^{\mathrm{er}}$.

«Synonyme, en première analyse, d'égalité en ce sens qu'il implique que le service public s'abstienne de privilégier un usager ou groupe d'usagers, la neutralité signifie, en seconde analyse, que le service public doit s'abstenir d'afficher sous quelque forme que ce soit, des convictions qui lui sont propres ${ }^{10}$.

«Les usagers doivent [donc] être traités de la même façon, quelles que puissent être leurs croyances religieuses ${ }^{11}$.

En effet, l'un des aspects essentiels de la neutralité est de ne faire aucune discrimination entre les usagers du service public en fonction de leurs opinions, politiques, religieuses, philosophiques, voire de leur rattachement ethnique.

1. A. Fossier, Les Manifestations cultuelles sur les voies publiques en France, Thèse, Paris, Spes, 1927, p. 92.

2. Discours du président de la République, M. Jacques Chirac, prononcé le 17 décembre 2003, suite au dépôt du Rapport Stasi, rendu public le 11 décembre précédent; cette phrase a été citée par J. Morange, « Peut-on réviser la loi de 1905 ? », RFDA, n 1, 2005, p. 156.

3. G. Lebreton, Libertés publiques et droits de l'homme, 6 éd., Paris, A. Colin, 2003, p. 399.

4. «La liberté des cultes est une des libertés fondamentales dont le principe a valeur constitutionnelle. Elle constitue, aujourd'hui, l'un des aspects de la liberté religieuse, avec la liberté de conscience. L'une et l'autre sont jugées inséparables. L'adhésion libre à une foi, la pratique religieuse individuelle (prière et comportement) sont indissociables de l'appartenance à une communauté et à une pratique collective, dont le culte constitue l'un des éléments » (G. Morange, Droits de l'homme et libertés publiques, $5^{\mathrm{e}}$ éd., Paris, PUF (Droit fondamental), 2000, p. 369, $\mathrm{n}^{\circ} 295$ ).

5. Ibid., p. 369-370, $\mathrm{n}^{\circ}$ 296. C'est pourquoi l'article 31 de la loi du 9 décembre 1905 prévoit une condamnation pénale à l'encontre de toute personne « [....] qui, soit par voies de fait, violences ou menaces contre un individu, soit en lui faisant craindre de perdre son emploi ou d'exposer à un dommage sa personne, sa famille ou sa fortune, l'auront déterminé à exercer ou à s'abstenir d'exercer un culte, à faire partie ou à cesser de faire partie d'une association cultuelle, à contribuer ou à s'abstenir de contribuer aux frais d'un culte».

6. J. Le Goff, «Approche comparée de la mise en œuvre du principe de neutralité politique par les juridictions administrative et judiciaire », in Convergences. Mélanges Marcel David, Quimper, Calligrammes, 1991, p. 288.

7. Voir V. Kondylis, Le Principe de neutralité dans la fonction publique, Paris, LGDJ (Bibliothèque de droit public; t. 168), 1994, p. 3-4.

8. C. Durand-Prinborgne, La Lä̈cité, Paris, Dalloz (Connaissance du droit), 1996, p. 47.

9. Voir, sur ce point, G.-J. Guglielmi et G. Koubi, Droit du service public, Paris, Montchrestien (Domat droit public), $2000, \mathrm{p} .443, \mathrm{n}^{\circ} 1136$.

10. S. Braconnier, Droit des services publics, Paris, PUF (Thémis droit public), 2003, p. 274.

11. B. Stasi (commission présidée par), Laïcité et République, Rapport au président de la République, Paris, La Documentation française, 2004 , p. 51. 
Dans un second sens, la neutralité s'identifie au terme d'impartialité $^{12}$ et signifie par là même l'abstention de l'État à prendre parti pour telle option, philosophique, politique ou religieuse, par exemple. «[...] l'État laïque admet toutes les religions, mais n'en reconnait aucune ${ }^{13}$.

«L'État ne peut prétendre assurer la sauvegarde de la liberté d'opinion que si le service public est neutre ${ }^{14}$.

Toutefois, «Il ne s'agit pas de parler de neutralité d'abstention, mais de neutralité de respect. Car si l'État laïque ne doit défendre aucun dogme religieux, il ne doit pas, pour autant, promouvoir une conviction athée ou agnostique. Il doit veiller, dans les relations avec les cultes et avec l'ensemble des familles spirituelles, à ce que tous puissent s'exprimer $[\ldots] »^{15}$.

C'est ainsi que l'État protège la liberté d'exercice des cultes le cas échéant, si celle-ci est contrariée.

Par suite, et particulièrement, à l'école, la neutralité, pas seulement religieuse au demeurant, vise à ce que l'enseignement ne favorise pas « [...] la propagation vers de jeunes élèves, particulièrement influençables, d'aucune doctrine religieuse ou politique ${ }^{16}$.

\section{b. L'impact de la neutralité religieuse sur les lieux publics}

La laïcité - c'est-à-dire la neutralité religieuse - s'impose aux lieux publics, et ce, parce qu'elle garantit la liberté de conscience des utilisateurs d'un tel espace, et par là même, celle de croire ou de ne pas croire.

C'est ainsi qu'elle impose, en principe, que ceux qui croient ne puissent pas importuner, par leur expression religieuse, et pire leur prosélytisme religieux, dans les lieux publics, ceux qui ne croient pas ou qui croient à une foi différente de la leur.

Le respect de la "liberté de conscience négative (le droit de ne pas croire) » ${ }^{17}$ vaut bien, en effet, celui de la "liberté de conscience positive (celui de croire librement)».

«Ces exigences de neutralité de l'État s'appliquent tout particulièrement au système scolaire, qui constitue sans doute depuis deux siècles le terrain le plus conflictuel en matière de laïcité de l'État ${ }^{18}$.
Dès lors, la neutralité religieuse des lieux publics impliquerait l'impossibilité d'y exprimer sa foi.

«Ainsi, la neutralité de l'État en matière cultuelle commande d'interdire, par exemple, la présence d'une croix ou d'un crucifix dans la salle d'audience d'un tribunal ou encore sur le mur d'une salle de classe dans une école publique ${ }^{19}$.

Hors des lieux publics, la laïcité n'empêche nullement de pouvoir exprimer voire de pratiquer librement sa foi, sans risque d'être empêché, ni discriminé pour cela. L'espace privé, le lieu d'épanouissement de la vie privée ${ }^{20}$, serait donc le lieu de la liberté d'expression religieuse.

En effet, et depuis la loi de séparation du 9 décembre 1905, « [...] non seulement l'État n'impose aucune conviction (absence de religion officielle), mais encore l'activité religieuse devient strictement privée ${ }^{21}$.

C'est pourquoi la jurisprudence administrative a toujours admis les manifestations individuelles du culte.

C'est ainsi qu'est soumise au principe de liberté l'apposition d'emblèmes religieux sur une maison privée ${ }^{22}$ ou le port d'un costume religieux ${ }^{23}$.

Il en va de même lorsqu'elles sont semi-collectives telles que les convois funèbres ${ }^{24}$.

D'ailleurs, « [...] la liberté de conscience est une condition fondamentale de l'existence d'une sphère privée de l'individu différente d'une sphère publique ${ }^{25}$.

C'est pourquoi, l'article $1^{\text {er }}$ de la loi du 9 décembre 1905 de séparation de l'Église et de l'État dispose que « La République assure la liberté de conscience. Elle garantit le libre exercice des cultes sous les seules restrictions édictées ci-après dans l'intérêt de l'ordre public».

L'État laïc est donc, hors de l'espace public, c'est-àdire dans les lieux privés, le garant de l'expression religieuse.

À l'égard de la liberté d'expression religieuse, l'État, selon son espace d'exercice, peut soit l'interdire, soit la protéger.

Ainsi, la «neutralité religieuse » «[...] place l'État dans la position d'un arbitre dont les règles du jeu sont les droits de l'homme», et ce, parce que « [...] c'est précisément pour être pluraliste que l'État doit être neutre ${ }^{26}$.

12. E. Mitard, «L'impartialité administrative », AJDA, 1999, p. 478-495.

13. C. Leclercq, Libertés publiques, $5^{\mathrm{e}}$ éd., Paris, Litec, 2003, p. $317, \mathrm{n}^{\circ} 426$.

14. J. Robert, avec la collaboration de J. Duffar, Droits de l'homme et libertés fondamentales, $7^{\mathrm{e}}$ éd., Paris, Montchrestien (Domat droit public), 1999 , p. 566.

15. B. Stasi (entretien avec), «La loi de 1905, pierre angulaire du pacte républicain », CFP, avril 2004, p. 17.

16. J.-F. Lachaume, H. Pauliat et C. Boiteau, Grands services publics, $3^{\mathrm{e}}$ éd., Paris, A. Colin (U), 2004, p. 436.

17. M. Onfray, Traité d'athéologie, Paris, Grasset, 2005.

18. L. Wauquiez-Motte, «Laïcité et neutralité de l'État», CFP, $\mathrm{n}^{\circ}$ 233, 2004, Les Personnes publiques face aux religions, p. 8.

19. P. Mabaka, «La laïcité, concept juridique ou philosophique?», Politéia, $\mathrm{n}^{\circ}$ 5, 2004, p. 397, $\mathrm{n}^{\circ} 15$.

20. G. Armand, L'Autorité judiciaire, gardienne de la liberté individuelle, dans la jurisprudence du Conseil constitutionnel, Thèse, Université de Caen BasseNormandie, 2000, p. 122-130.

21. J. Fialaire et É. Mondielli, Droits fondamentaux et libertés publiques, Paris, Ellipses, 2005, p. 465.

22. M. Hauriou, Précis de droit administratif et de droit public [1892], 12 éd., Paris, Sirey, 1933 (mis à jour par A. Hauriou) (réédition présentée par P. Delvolvé et F. Moderne, Paris, Dalloz, 2002), p. 641.

23. CE 19 mars 1909, Abbé Deguille, Rec. 307 (concl. Saint-Paul), S., 1909. III. 99 ; CE 2 août 1907, Abbé Garcin, Rec. 756, S., 1909. III. 1 (note M. Hauriou); CE 14 février 1908, Abbé Valette, Rec. 143, S., 1909. I. 3 (note M. Hauriou) ; CE 19 février 1909, Abbé Olivier, Rec. 181, D., 1910, p. 121 (concl. Chardenet), RDP, 1910, p. 69 (note G. Jèze); CE 14 mai 1915, Buléon, Rec. 159; CE 24 décembre 1915, Abbé Chaumontet, Rec. 368.

24. CE 19 février 1909, Abbé Olivier, Rec. 181, S., 1909. III. 34 (concl. Chardenet), D., 1910. III. 121 (concl.), RDP, 1910, p. 69 (note G. Jèze).

25. G. Koubi, «Autorisations d'absence et liberté de conscience des fonctionnaires », RA, 1987, p. 134.

26. O. Schrameck, «Laïcité, neutralité et pluralisme», in Mélanges Jacques Robert, Paris, Montchrestien, 1998, p. 204. 
Mais, avant d'aller plus loin dans cette réflexion, il importe de s'entendre sur la notion même de «lieux publics », au-delà des quelques exemples susmentionnés.

\section{B. La notion de « lieu public »}

Définir la notion de «lieu public », formule utilisée en droit mais tirée plus du langage commun que de la terminologie juridique, est plus difficile à cerner qu'il n'y paraît.

Il importe donc d'évoquer son double sens (1), avant d'envisager ses rapports avec tant la distinction domaniale (2) que le « lieu d'exécution du service public» (3).

\section{Le double sens du « lieu public »}

La notion de «lieu public» peut s'entendre de deux manières.

Tout d'abord, et dans un sens étroit, elle peut ne viser que les lieux - au sens exclusif d'immeubles - appartenant aux personnes publiques. En somme, les lieux dits communément publics dans le langage administratif seraient finalement des immeubles d'appropriation publique, faisant l'objet d'un droit de propriété publique ${ }^{27}$.

Ensuite, et dans un sens large, voire excessivement large, le groupe de mots « lieu public » peut aussi se définir comme «tout lieu ouvert au public, recevant du public ${ }^{28}$. C'est dans ce sens que l'entend, par exemple, la loi $\mathrm{n}^{0}$ 2005-102 du 11 février 2005, pour l'égalité des droits et des chances, la participation et la citoyenneté des personnes handicapées.

Par suite, dans ce texte, est un lieu public tant un centre commercial ou un cinéma, d'appropriation pourtant privée, qu'un hôpital, une bibliothèque, ou encore, un théâtre, détenus par une personne publique. Finalement, il convient de souligner que l'adjectif «public », utilisé dans la loi et accolé au mot «lieu»-selon une formulation usuelle du langage courant -, l'est plus au sens commun qu'au sens strictement juridique.

Le «lieu public » aux prises avec la neutralité religieuse ne peut être naturellement que l'immeuble d'appropriation publique, dans la mesure où, comme cela a été pré- cédemment précisé, la sphère privée échappe normalement à l'application de la laïcité.

Mais, est-ce qu'une telle définition est exhaustive?

\section{Le " lieu public » et la distinction domaniale}

Reste toutefois à savoir si toutes les propriétés publiques immobilières font l'objet de l'application d'une telle règle, et sont soumises au respect de la neutralité religieuse.

La question mérite d'autant plus d'être posée que les biens des personnes publiques sont classés en deux domaines : le domaine public et le domaine privé, sachant que le second fait essentiellement ${ }^{29}$ l'objet d'une définition négative, posée par l'article L 2 du Code du domaine de l'État.

Quant au domaine public, il se définit comme un ensemble de biens, immobiliers voire - plus rarement mobiliers, appartenant à une personne publique et ainsi qualifié de façon soit textuelle, soit naturelle ${ }^{30}$, soit jurisprudentielle.

Sachant que, sur le plan jurisprudentiel, un bien ne constitue un domaine public qu'à la double condition d'être affecté à l'usage de tous ${ }^{31}$ ou d'un service public ${ }^{32}$ et d'être spécialement aménagé à cet effet ${ }^{33}$.

Dans la mesure où, d'une part la gestion du domaine privé ne constitue pas un service public ${ }^{34}$, et ce, malgré les critiques doctrinales ${ }^{35}$, et que, d'autre part, la neutralité religieuse se rattache avant tout à la notion de service public, ce principe semble difficilement applicable aux immeubles appartenant à des personnes publiques et qualifiés de domaine privé.

Par conséquent, il apparaît que le principe de neutralité se rattache plus facilement au domaine public qu'au domaine privé.

Toutefois, rien n'est moins évident, particulièrement lorsque le domaine public fait l'objet d'une occupation privative; ainsi, en est-il, par exemple, des concessions funéraires, qui permettent à leurs occupants d'exprimer leur liberté religieuse.

Cette difficulté à associer de manière précise la neutralité religieuse à une catégorie de biens précise tient peut être tout simplement au fait que la laïcité n'est pas tant une

27. Sur le droit de propriété publique, voir P. Yolka, La Propriété publique. Éléments pour une théorie, Paris, LGDJ (Bibliothèque de droit public; t. 191), 1997 ; H. Moysan, Le Droit de propriété des personnes publiques, Paris, LGDJ (Bibliothèque de droit public; t. 219 ), 2001.

28. À cet égard, la jurisprudence criminelle qualifie «l'école publique [comme étant] un lieu public par destination » (Cass. civ. $2^{\mathrm{e}} 3$ juillet $2003, X . . . c$. TI Lorient, D. Pén. 11 / 2003, nº 118 (note M. Véron)).

29. À noter toutefois que, depuis l'ordonnance n ${ }^{\circ} 2004-825$ du 19 août 2004, le nouvel alinéa 2 de l'article L 2 du Code du domaine de l'État est ainsi complété : «[...] les biens immobiliers à usage de bureaux, propriété de l'État ou de ses établissements publics, à l'exclusion de ceux formant un ensemble indivisible avec des biens immobiliers appartenant au domaine public, font partie du domaine privé de ces personnes publiques.» Ce qui inaugure une ébauche de définition positive du domaine privé, contraire au demeurant aux critères jurisprudentiels d'identification du domaine public.

30. Cette qualification est aussi, mais indirectement, fondée sur un texte car tout domaine public naturel (maritime ou fluvial, par exemple) est, en principe, en ses composantes, défini par des textes.

31. CE Sect. 28 juin 1935, Marecar, Rec. 734, RDP, 1935, p. 590 (concl.; note G. J.), D., 1936. III. 20 (concl. R. Latournerie; note M. Waline), S., 1937. III. 43 (concl.), Lachaume p. 425.

32. CE Sect. 19 octobre 1956, Société Le Béton, Rec. 375, AJDA, 1956, 2, p. 472 (concl. M. Long) et p. 488 (ch. J. Fournier et G. Braibant), D., 1956, p. 681 (concl.), RA, 1956, p. 617 (note G. Liet-Veaux), RA, 1957, p. 131 (note R. Morice; note G. Liet-Veaux), RDP, 1957, p. 310 (concl.), JCP, 1957. II. nº 9765 (obs. C. Blaevoet), GAJA, nº 89 .

33. CE Ass. 11 mai 1959, Dauphin, Rec. 294, AJDA, 1959, 1, p. 113 (ch. M. Combarnous et J.-M. Galabert) et, 2, p. 228 (note J. Dufau), D., 1959 , p. 314 (concl. H. Mayras), JCP, 1959. II. nº 11269 (obs. J. de Lanversin), S., 1959, p. 117 (concl.), Lachaume p. 426; CE Ass. 22 avril 1960, Berthier, Rec. 264, AJDA, 1960. I. 78 (ch. M. Combarnous et J.-M. Galabert) et 140 (note P. Vergnaud), RDP, 196o, p. 1213 (concl. J.-F. Henry).

34. D.-G. Lavroff, Le Régime du domaine privé, Répertoire des collectivités locales, Dalloz, fascicule 5042, p. 13-14, $\mathrm{n}^{\circ} 85-86$.

35. Par exemple J.-M. Auby, «Contribution à l'étude du domaine privé de l'administration », EDCE, nº 12, 1958, p. 35-57. 
caractéristique du régime juridique des biens des personnes publiques mais une loi du service public.

Par suite, c'est finalement au regard de l'activité gérée, et par là même moins au regard du statut juridique du bien utilisé pour l'exercer, que s'applique la neutralité religieuse.

\section{Le « lieu public » et l'exécution du service public}

«Il résulte des textes constitutionnels et législatifs que le principe de liberté de conscience ainsi que celui de la laïcité de l'État et de neutralité des services publics s'appliquent à l'ensemble de ceux-ci ${ }^{36}$. Ce qui sous-entend : «quel que soit le gestionnaire du service public considéré».

En théorie, les activités qualifiées de services publics, qu'elles soient gérées par des personnes publiques ou par des personnes privées, sont en principe soumises aux trois lois - traditionnelles - du service public que sont la continuité, l'égalité et la mutabilité.

Ce qui toutefois pose un problème actuel au regard des caractéristiques du «lieu d'exécution des services publics».

En effet, aujourd'hui, de plus en plus, les personnes publiques non seulement délèguent la gestion de leurs services publics, mais aussi vendent leurs propriétés immobilières et louent - le plus souvent - les bâtiments d'exécution de ces services voire achètent pour ce faire des appartements dans des immeubles en copropriété privée $^{37}$.

Ceci est d'autant plus problématique au regard de l'application de la neutralité religieuse que le juge administratif considère que la domanialité publique est incompatible avec les règles de la copropriété privée ${ }^{38}$.

Peut-on alors considérer que la neutralité du service public est violée lorsqu'une commune loue ou achète, pour une annexe du service d'état civil par exemple, un appartement dans un immeuble et que dans les parties communes de ce bâtiment sont apposés des symboles religieux?

Quelle sera alors l'attitude du juge administratif, saisi par des usagers, non croyants ou de croyance opposée à de tels symboles, et qui devront emprunter ces parties communes afin de se rendre au bureau où ils bénéficieront de prestations de service public?

De plus, en déléguant largement leurs services publics, les personnes publiques prennent le risque de voir ces activités d'intérêt général réalisées dans des lieux privés alors même que rares sont les contrats de délégation de service public rappelant le respect dû à la neutralité religieuse.
De surcroît, qui ira vérifier que les locaux du délégataire privé sont complètement neutres religieusement voire politiquement?

Pourtant, un grand nombre de lieux privés sont le siège d'exécution de services publics essentiels tels que les établissements d'enseignement privé associé à l'exercice du service public de l'Éducation nationale. De par le « caractère propre » des conditions de l'enseignement qui y est dispensé, et ce, malgré le respect par les enseignants des programmes nationaux de l'Éducation nationale, ces établissements, bien qu'associés, parfois grandement, à ce service public, n'en respectent pas toutes les lois, dont celle, incidente, de la neutralité religieuse; sachant que s'ils le faisaient, ils nieraient leur spécificité.

Pourtant, dans le cadre de la discussion de la loi $\mathrm{n}^{\circ}$ 2004-228 du 15 mars 2004 encadrant, en application du principe de laïcité, le port de signes ou de tenues manifestant une appartenance religieuse dans les écoles, collèges et lycées publics, qui a interdit, dans tous les établissements primaires et secondaires, le port de tous signes " ostensibles» d'appartenance religieuse (article L 141-5-1 du Code de l'éducation), l'actuel président de l'Assemblée nationale, M. Jean-Louis Debré, souhaitait étendre le champ d'application de cette interdiction aux établissements d'enseignement privé ${ }^{39}$.

D’ailleurs, les établissements privés confessionnels, associés au service public de l'Éducation nationale, ne retirent pas le crucifix, éventuellement apposé au mur des salles de cours, lorsque ces dernières sont transformées en salles d'examen pour le déroulement des épreuves du baccalauréat.

Certes, la jurisprudence administrative a surtout eu à connaître des affaires en matière de neutralité religieuse où étaient principalement, pour ne pas dire exclusivement, en cause des immeubles constitutifs de domaine public.

Mais, n'est-ce pas parce que jusqu'à présent la neutralité religieuse n'a posé problème quant à son application qu'au regard de certains services publics, tel qu'en particulier celui de l'Éducation nationale, et que ces services sont exercés dans des immeubles domaniaux publics?

Qu'en sera-t-il demain lorsque des écoles seront construites dans le cadre de contrats de partenariat ou de baux emphytéotiques administratifs et que les personnes publiques n'en seront donc pas propriétaires immédiatement voire jamais en fonction de leurs intentions?

Ces défis d'avenir de la neutralité religieuse des lieux publics ne peuvent qu'amener à s'interroger sur les enseignements tirés des « leçons » du passé.

36. CE 3 mai 2000, Avis ${ }^{\circ} 217017, M^{\text {lle }}$ Marteaux, Rec. 169, AJDA, 2000, p. 602 (ch. M. Guyomar et P. Collin), RFDA, 2000, p. 899, DA, 2000, nº 189 (note R. S.), RFDA, 2001, p. 146 (concl. R. Schwartz), D., 2000, p. 747 (note G. Koubi), RRJ, 2001-4 (II), p. 2107 (note G. Armand).

37. P. Yolka, «Un État sans domaine public», AJDA, $\mathrm{n}^{\circ} 20,2003$, p. 1017.

38. CAA Paris 20 juin 1989, Ministre de l'Économie et des Finances c. Société La Préservatrice foncière, Rec. 321, DA, 1989, nº 560, CJEG, 1989, p. 362 (note P. Sablière) et p. 368, JCP, 1990. II. nº 21506 (note J.-P. Davignon), RJTC, 1990, p. 227, RDP, 1990, p. 545 (note F. Llorens); CE 11 février 1994, Compagnie d'assurances La Préservatrice foncière, Rec. 64, AJDA, 1994, p. 548 (note J. Dufau), CJEG, 1994, p. 197 (ch. P. Sablière) et p. 208 (concl. H. Toutée), D., 1994, p. 493 (note J.-F. Davignon), JCP, 1994. II. nº 22338 (note M.-C. Rouault), RFDA, 1994, p. 501 (concl.), DA, 1994, nº 196, MTPB, 4 mars 1994, p. 37 Répertoire Défrenois, 1995, p. 490 (note O. Farthouat).

39. Le Figaro, 10 octobre 2003. 


\section{L'histoire de la neutralité religieuse des lieux publics}

«La laïcité est un phénomène singulièrement lié à l'histoire de la France, l'instauration de sa Constitution et le développement de ses institutions ${ }^{40}$.

\section{La période précédant le vote de la loi du 9 décembre 1905}

Sous l'Ancien Régime, la monarchie est donc de droit divin et le Roi est le représentant de Dieu sur Terre.

C'est en réaction contre cette situation que se conçoit le contenu de l'article 10 de la Déclaration des droits de l'homme et du citoyen du 26 août 1789 , qui proclame la liberté de conscience. Dès lors, et depuis la Révolution française, la question, jusqu'alors ignorée, de la neutralité religieuse des lieux publics voire des lieux cultuels s'est imposée.

Le 2 novembre 1789, les biens du clergé sont mis à la disposition de la Nation, sans indemnité. Plus la Révolution avance, et surtout, s'enfonce dans la guerre civile, plus la méfiance des révolutionnaires à l'égard du catholicisme augmente. «Avec la Convention, la persécution religieuse atteignit son paroxysme ${ }^{41}$.

«Tous les lieux de culte catholique étaient fermés; quiconque en redemandait l'ouverture, arrêté comme suspect et proscrit. Le culte catholique, quel qu'il soit, intérieur et extérieur, avait complètement disparu ${ }^{42}$.

Le Directoire instaure alors, par l'article 354 de la Constitution du 22 août 1795, une stricte séparation des Églises et de l'État s'interprétant en une ignorance du pouvoir spirituel par le pouvoir temporel.

Cela n'empêche toutefois pas ce même régime politique d'interdire toute manifestation extérieure du culte, y compris les sonneries de cloches appelant les fidèles à la messe! Même si, finalement, la liberté des cultes connut alors une certaine effectivité.

D'ailleurs, "À partir de l'an VIII et durant tout le $\left[\mathrm{XIX}^{\mathrm{e}}\right]$ siècle, le principe de la liberté posé à l'article $10 \mathrm{de}$ la Déclaration de 1789 n'a plus jamais été remis en question. Il est allé de pair avec le principe d'égalité civile ${ }^{43}$.

Napoléon, soucieux de soustraire les catholiques à l'influence des monarchistes, s'attire les bonnes grâces de la Papauté, en négociant avec le pape un Concordat, conclu en 1801. Il y est prévu d'ailleurs, dès l'article $1^{\mathrm{er}}$, la possibilité d'exercer librement son culte: «La religion catholique, apostolique et romaine, sera librement exercée en France et son culte sera public, en se conformant aux règlements de police que le gouvernement jugera nécessaires pour la tranquillité publique. »

C'est la police administrative qui est la borne de la manifestation religieuse ; malgré cela, la liberté religieuse peut à nouveau s'exprimer, et ce, même dans les lieux publics.

Ce système existe toujours en Alsace-Lorraine ${ }^{44}$ et en Guyane. Mais, il a disparu dans les autres territoires français régis, quant à eux, par la loi du 9 décembre 1905 sur la séparation de l'Église et de l'État. C'est pourquoi les développements postérieurs ne concernent que le territoire national soumis à l'empire de la loi de 1905.

\section{La loi du 9 décembre 1905}

La loi de 1905 a tenté de clore le sujet en renvoyant chaque pouvoir dans son domaine naturel de compétences. Mais, c'était oublié qu'un grand nombre de questions religieuses interfèrent, même malgré elles, dans le domaine du temporel ; ainsi en est-il, par exemple, du statut juridique des édifices cultuels et des cimetières.

Sous la III ${ }^{e}$ République, avec la loi de séparation, la question de la neutralité religieuse des lieux publics est incidemment réapparue, dans la mesure où la laïcisation de la sphère publique supposait, pour ne pas dire imposait, la réalisation de cette neutralité, accessoire finalement indissociable de la neutralité du service public.

C'est ainsi qu'au nom de cette neutralité, certains « lieux publics » ont alors été laïcisés tels que:

- les cimetières: suppression des carrés confessionnels par les lois du 14 novembre 1881 et du 5 avril 1884;

- le Parlement: suppression de la prière publique à la rentrée parlementaire par la loi du 14 août 1884 ;

- les établissements scolaires et autres bâtiments publics : interdiction des emblèmes religieux ${ }^{45}$ et des crucifix ${ }^{46}$.

\section{Les rencontres des deux pouvoirs dans les lieux publics}

Une société donnée, et a fortiori un État, ne peuvent jamais véritablement être neutres, ni même indifférents à l'égard de la religion.

En effet, si la laïcité de l'État correspond à la notion de «neutralité religieuse» avec comme conséquence « une ignorance de la part de l'État du fait religieux qui appartient à la sphère privée de chaque individu », " cependant, [...] l'ignorance ne peut être totale au risque $[. .$.

40. D. Fairgriewe, «Un État non laïc : le Royaume-Uni », CFP, nº 217, 2002, La Laïcité, p. 15.

41. A. Fossier, Les Manifestations cultuelles..., p. 11.

42. Ibid.

43. J. Morange, «Peut-on réviser la loi de 1905 ?», p. 154

44. J.-F. Flauss, «Droit local alsacien-mosellan et Constitution», RDP, 1992, p. 1625-1685; J. Miet, «Le droit local des cultes en Alsace-Moselle», CFP, $\mathrm{n}^{\circ} 233,2004$, Les Personnes publiques face aux religions, p. 14-16.

45. Article 28 de la loi du 9 décembre 1905.

46. Comme l'explique fort bien A. Le Goff, dans sa thèse, La Neutralité religieuse de l'État et l'école publique en France et en Allemagne (Université Panthéon-Sorbonne - Paris I, 2003, p. 347-349), il n'existe pas de fondement juridique explicite à ce retrait sauf à l'interpréter comme une conséquence logique de la loi du 26 mars 1882 instituant, en France, la laïcité de l'enseignement primaire. 
de remettre en cause [...] la possibilité pour les individus de pratiquer leur liberté religieuse $[\ldots] »^{47}$.

Qu'il soit religieux, areligieux ou irreligieux, cet État se détermine toujours par rapport à la religion, en l'acceptant ou en la rejetant voire en l'ignorant!

C'est pourquoi «[...] plusieurs exemples montrent que les relations entre l'État et les religions ont évolué dans le sens du dialogue et même parfois de la coopération. Ainsi, le service public de radio (France-Culture) et de télévision (actuellement France 2) doit diffuser le dimanche matin des émissions religieuses au profit des cultes juif, protestant, catholique et musulman. Ces quatre religions sont même représentées dans des organismes consultatifs chargés de donner leur avis sur des problèmes moraux précis ayant une dimension sociale, comme le Comité consultatif national d'éthique créé en 1983 et le Conseil national du sida créé en $1989{ }^{48}$.

Dès lors, une vision pluraliste du monde, en matière religieuse ainsi, finalement, qu'en toute matière, est peut être plus conforme aux sociétés actuelles.

«Il reste que la neutralité de la prestation de service public et son environnement ne peut plus faire aujourd'hui abstraction d'un certain nombre de données issues de la volonté de ne pas couper l'école de la société et donc de la diversité de celle-ci et il est quelquefois difficile de combiner neutralité et "revendications identitaires", susceptibles à leur tour de soulever des problèmes sur le plan de la sécurité dans le fonctionnement du service public ${ }^{49}$.

Si intégrer signifie uniformiser de peur de voir émerger une société "communautariste», cela signifie aussi essayer de gommer toutes les différences sociales, religieuses, intellectuelles, etc., cela revient à faire fi de la réalité en se tournant vers un individu idéal, un étalon-individu aussi aseptisé que certains produits vendus sous vide en grande surface.

Par suite, et au regard des aménagements financiers voire fiscaux, de la possibilité d'ouvrir des aumôneries dans les prisons, les hôpitaux voire les établissements scolaires avec internat, de certaines tolérances administratives à l'égard des carrés confessionnels, « Les exigences d'une neutralité absolue sont donc tempérées par les "accommodements raisonnables" permettant à chacun d'exercer sa liberté religieuse ${ }^{50}$.

En somme, «[...] la neutralité cultuelle de l'État n'équivaut pas à l'indifférence ou incompétence des autorités publiques envers les questions religieuses ou spirituelles ${ }^{51}$, il apparaît avec une certaine évidence que la neutralité religieuse affirmée est plus nuancée qu'on ne peut le penser dans la réalité administrative.

\section{E. L'annonce de plan}

Il apparaît avec évidence que l'État républicain s'est construit dans le respect des différences religieuses en faisant de la laïcité un de ses piliers fondateurs, prolongement du principe d'égalité.

Cette affirmation du respect de toutes les croyances par l'État, et par prolongement par toutes les personnes publiques, vise à éviter tout traitement discriminatoire à l'égard des administrés voire des usagers. Ce qui implique à la charge des agents publics le respect d'un devoir de neutralité ${ }^{52}$. En effet, le lieu public n'est effectivement neutre que si, et surtout, les « agents du service public» le sont.

Toutefois, la rigueur du principe de la neutralité religieuse des lieux publics apparaît bien théorique au regard de certains lieux et, finalement, s'atténue en fonction des lieux publics considérés.

Ainsi en est-il pour les lieux consacrés comme les édifices cultuels et les cimetières, mais aussi, pour ceux qui sont tolérés par la puissance publique, au sein même de lieux publics en vue d'assurer le respect de l'expression de la liberté religieuse, à l'instar des aumôneries.

C'est pourquoi, en soi comme au regard du statut des agents publics, la neutralité religieuse s'impose - finalement de manière plus traditionnelle que strictement juridique - comme une obligation de service public (I).

Mais, les personnes publiques ne peuvent pas totalement rester indifférentes aux questions religieuses au regard des faits (affaires dites du "foulard islamique ", rites et processions religieuses sur la voie publique, etc.).

Par suite, si elles ne doivent pas se mêler des concepts et dogmes religieux, elles ne peuvent ignorer la gestion des affaires touchant aux religions.

Dès lors, et en fonction de la spécificité de certains lieux publics, la neutralité officiellement affirmée a parfois tendance, dans son application à ces lieux, à s'atténuer en rigueur (II).

\section{La neutralité religieuse dans les lieux publics : une obligation affirmée}

La neutralité religieuse, à l'instar de la neutralité politique $^{53}$, a toujours été affirmée dans le cadre du service public.

47. Ibid., p. 19 .

48. Ibid., p. 23. Voir aussi J.-M. Pontier, Droits fondamentaux et libertés fondamentales, $2^{\mathrm{e}}$ éd., Paris, Hachette (Les Fondamentaux), 2005, p. 102.

49. J.-F. Lachaume, H. Pauliat et C. Boiteau, Grands services publics, p. 436.

50. B. Stasi (commission présidée par), Laïcité et République, Rapport au président de la République, p. 54.

51. P. Mabaka, «La laïcité, concept juridique ou philosophique?», p. 397, nº 15.

52. E. Aubin, Droit de la fonction publique, $2^{\mathrm{e}}$ éd., Paris, Gualino (Mémentos), 2004, p. 191-194; J.-M. Auby, J.-B. Auby, D. Jean-Pierre et A. Taillefait, Droit de la fonction publique, $4^{\mathrm{e}}$ éd., Paris, Dalloz, 2002, p. 300-303 ( $\mathrm{n}^{\circ}$ 603-606); T. Ben Salah, Droit de la fonction publique, $2^{\mathrm{e}}$ éd., Paris, A. Colin (Compact), 2003, p. 189-19o ( $\mathrm{n}^{\mathrm{o}} 266$ ) ; J.-F. Lachaume, La Fonction publique, $3^{\mathrm{e}}$ éd., Paris, Dalloz (Connaissance du droit), 2002, p. 87-90.

53. «La neutralité politique des bureaux de vote (c'est-à-dire celles et ceux qui les constituent) et des salles de vote (c'est-à-dire le local) (est présentée comme) une garantie de la sincérité du scrutin » (B. Maligner, note sous CE 29 novembre 2004, Élections municipales de Contrevoz, AJDA, $\mathrm{n}^{\circ} 8$, 2005, p. 438). Voir sur la même question: CE 8 mars 2002, Élections municipales de la commune de Vairoo et M. Doom, Rec. 92, AJDA, 2002, p. 1014 (ch. F. Donnat et D. Casas), DA, 2002, nº 142 (obs. C. Maugüé); CE 15 novembre 2004, Élections à l'Assemblée de Polynésie française, req. $\mathrm{n}^{\circ} 268.543$. 
En effet, «Le principe de neutralité du service public est d'abord une garantie de l'existence "administrative" des services ; s'il est considéré comme le seul frein à une politisation excessive des activités de service public, il devrait également être un moyen de rendre compte d'un principe d'indifférence des services à l'égard de toute question de nature religieuse. Cependant, s'il s'agit d'éviter tout risque de discrimination ou de partialité à l'égard des participants ou des usagers à / de tout service public, ce principe est aussi la voie par laquelle s'annonce la prise en compte des différenciations sociales ${ }^{54}$.

Dès lors, la neutralité religieuse dans les lieux publics, cadres traditionnels d'exécution des services publics, s'appuie sur une justification essentielle : la volonté, dans le cadre de la gestion de ces lieux et de leur accès aux usagers des services publics, de ne pas les discriminer à raison de leur croyance ou de leur non-croyance. Ce qui ne signifie pas toutefois que les usagers du service public ne connaissent aucune borne à l'expression de leur liberté religieuse dans les lieux publics, bien au contraire (A).

Mais, et dans la mesure où un lieu public n'est qu'un cadre d'évolution et d'action des agents publics, des "serviteurs »- au sens noble du terme ${ }^{55}$ - du service public, il convient pour que ce lieu soit neutre que ces agents le soient. La neutralité religieuse des agents publics, et par là même le devoir de réserve auquel ils sont astreints, sont des conditions sine qua non de la neutralité du lieu public (B).

\section{A. Un principe général: l'obligation de neutralité religieuse}

\section{Une justification essentielle :} la non-discrimination religieuse

«On retrouve dans la plupart des pays d'Europe, un ensemble de droits spécifiques des usagers des services collectifs [tels que : 1'] égalité des usagers devant le service public qui se décline en fait en deux principes : égalité d'accès; égalité de traitement ${ }^{56}$.

D'ailleurs, dans le traité portant projet de Constitution européenne, parmi les valeurs de l'Union européenne, sont mentionnées la «non-discrimination» et «l'égalité entre les femmes et les hommes» (art. I-2).

«Le principe d'égalité est la source du principe de non-discrimination entre les individus, quels que soient leur origine, religion, race, sexe, etc. ${ }^{57}$. Ce que confirme l'article $1^{\text {er }}$ de la Constitution du 4 octobre 1958 et que protège l'article 225-1 du Code pénal. Ce principe de nondiscrimination est aussi rappelé par la loi $\mathrm{n}^{0}$ 2005-158 du
23 février 2005, portant reconnaissance et contribution nationale en faveur des français rapatriés, en son article 5, au profit des harkis.

Ce principe signifie donc que certaines distinctions sont strictement interdites et ne peuvent donc pas justifier une quelconque distinction de traitement entre les usagers du service public.

Alors que des critères relatifs à la situation géographique des usagers ou à leur situation sociale et financière peuvent fonder des traitements distincts d'usagers. En effet, «[...] l'application du principe d'égalité n'a pas pour effet de supprimer les disparités, ni de nier la diversité ${ }^{58}$.

En somme, «Le principe d'égalité [...] n'implique plus nécessairement l'uniformité de la législation et n'impose plus de traiter tous les individus de façon identique ; il s'interprète comme un principe de non-discrimination, c'est-à-dire qu'il proscrit seulement les différences de traitement arbitraires, illégitimes, celles qui ne sont pas rationnellement justifiées par des différences de situation ${ }^{59}$.

Ainsi, la neutralité des lieux publics implique l'égal accès des usagers à ces biens, et par là même, leur libre et, en principe, gratuit usage, principes issus du droit administratif des biens ${ }^{60}$.

En effet, cette matière de droit administratif spécial enseigne relativement à la qualification d'un bien, appartenant à une personne publique, en vue de le qualifier de domaine public, que le bien d'appropriation publique, pour devenir un tel domaine, doit être affecté à l'usage de tous ou à l'exécution d'un service public, et incidemment spécialement aménagé à cet effet.

Par suite, le bien public qualifié aussi de domaine public se doit d'être accessible, et à cet effet, il doit être ouvert aux administrés.

C'est ainsi que pour accompagner un ami jusqu'à son train, une personne peut entrer dans une gare, même sans être usager du service public ferroviaire.

De même, «Tous les fidèles [...] ont une même aptitude légale à pénétrer dans les [lieux de culte], comme tous les promeneurs ont une même aptitude à entrer dans un jardin public ${ }^{61}$.

Certes, l'accès partiel à certains lieux publics, tels que les écoles, peut être fermement réglementé, le plus souvent d'ailleurs pour des raisons de sécurité.

Ces règles issues du droit administratif des biens ne peuvent que conforter celle de la neutralité, particulièrement religieuse, des lieux publics, se rattachant quant à elle plutôt au droit des services publics.

54. G.-J. Guglielmi et G. Koubi, Droit du service public, p. 443, ${ }^{\circ} 1136$

55. P. d'Iribarne, La Logique de l'honneur, Paris, Seuil, 1989.

56. J.-F. Auby, Les Services publics en Europe, Paris, PUF (Que sais-je?; $n^{\circ}$ 3414), 1998, p. 64

57. G.-J. Guglielmi et G. Koubi, Droit du service public, p. 382, $\mathrm{n}^{\circ} 984$.

58. Ibid., p. 396, $\mathrm{n}^{\circ} 1023$.

59. D. Lochak, «L'autre saisi par le droit », in L'Autre. Études réunies pour Alfred Grosser, B. Badie et M. Sadoun (dir.), Paris, Presses de la Fondation nationale des sciences politiques, 1996, p. 191.

60. J. Morand-Deviller, Cours de droit administratif des biens, $3^{\mathrm{e}}$ éd., Paris, Montchrestien, 2003, p. 240-256.

61. A. Mestre, note sous Cass. civ. 5 février 1912, Colonna et Abbé Forcioli, S., 1912. I. 354. 
En effet, l'accès libre des administrés aux lieux publics, éventuellement sièges d'exécution de services publics, suppose, pour que la conscience de ces utilisateurs ne soit pas heurtée, blessée, et par là même préservée, qu'ils soient autant que faire se peut neutres.

C'est pourquoi les symboles religieux, tels que les crucifix, doivent être retirés de tout bâtiment d'exécution d'un service public, en vertu de deux circulaires du 2 novembre 1882 et du 9 avril 1903 .

Ainsi, « [...] l'aménagement du cadre (c'est-à-dire les locaux scolaires) dans lequel sont dispensés les enseignements peut remettre en cause la neutralité religieuse de l'enseignement reçu dont le contenu en lui-même serait pourtant neutre ${ }^{62}$.

De surcroît, la loi du 9 décembre 1905, en son article 28 , interdit que soient apposés sur les monuments publics des emblèmes religieux.

Par suite, «l'apposition d'un emblème religieux postérieurement à l'entrée en vigueur de la loi de 1905, à l'extérieur comme à l'intérieur d'un édifice public communal, méconnaît à la fois la liberté de conscience, assurée à tous les citoyens de la République, et la neutralité du service public à l'égard des cultes quelconques ${ }^{63}$.

Le refus principiel connaît toutefois, dans le texte de 1905, diverses exceptions telles que les sépultures et monuments funéraires, les musées ou les expositions.

De plus, le juge administratif a grandement «neutralisé » cette disposition en tolérant, par exemple, que des cérémonies religieuses soient organisées à l'intérieur de bâtiments publics, à partir du moment où ceux-ci sont dotés d'aumôneries ${ }^{64}$.

C'est toutefois par respect pour le principe de neutralité du domaine public qu'a été justifiée, au nom de l'intérêt général, la discrimination opérée par une délibération votée par un conseil municipal. Aux termes de cette dernière, était interdite la location d'une salle des fêtes à tout groupement ou organisme à caractère politique ou religieux, et ce, afin d'éviter que l'utilisation de cette salle soit sujette aux querelles politiques et / ou religieuses ${ }^{65}$.

\section{Un exemple classique:}

les manifestations religieuses sur la voie publique

Les manifestations religieuses, telles que les processions religieuses, sont plus facilement en opposition avec les nécessités de la circulation donc de la sécurité publique parce qu'elles se déroulent le plus souvent sur la voie publique.
Comme tout lieu public, cette dernière doit respecter la neutralité religieuse ; dès lors, la police administrative municipale peut donc les réglementer voire interdire de telles manifestations extérieures du culte.

Mais, là encore, le juge administratif veille ${ }^{66}$ !

Les cérémonies extérieures sont le plus souvent traditionnelles et consacrent en quelque sorte l'universalité d'un culte. Ainsi illustrent-elles le fait qu'un culte religieux est rendu en tous temps et en tous lieux, et nullement renfermé dans la circonscription d'un lieu de culte. Ce qui finalement contredit cette volonté de la laïcité de vouloir systématiquement rejeter dans la sphère privée la religion et sa pratique.

D'ailleurs, « [...], il n'est pas contraire à l'esprit de la loi [de 1905] qu'un culte possédant des lieux de culte ajoute, aux cérémonies célébrées à l'intérieur de ces lieux, d'autres cérémonies accessoires qui se déroulent sur la voie publique ou qui, du moins, s'extériorisent d'un façon ou d'une autre. Ces cérémonies extérieures sont traditionnelles dans le culte catholique $»{ }^{67}$.

De plus, il importe de souligner que la neutralité de la voie publique est en aucune façon une finalité composant l'ordre public, et par là même, justifiant la mise en œuvre de cette police administrative.

Avant la Première Guerre mondiale, le juge administratif a parfois manqué de courage en n'annulant pas des arrêtés municipaux d'interdiction injustifiée de telles processions ${ }^{68}$; mais dès la veille de cette guerre ${ }^{69}$, il a fait preuve d'une grande sévérité à l'égard des errements administratifs laïcistes, sévérité dont il ne s'est plus départie depuis lors.

Aujourd'hui, "Les manifestations et cérémonies religieuses qui se déroulent hors des édifices cultuels (processions en particulier) sont soumises à déclaration préalable au maire. Mais, importante exception, cette déclaration n'est pas exigée si elles sont conformes aux usages locaux ${ }^{70}$.

En effet, la jurisprudence administrative est désormais claire : les processions traditionnelles sont systématiquement défendues et toute mesure de police les contrariant est, en principe, vouée à l'annulation ${ }^{71}$.

L'ancienneté de ces processions est un gage, en quelque sorte, de leur légalité. «Dans les cas des manifestations traditionnelles, la liberté et l'ordre public sont d'accord pour justifier leur maintien et la répression des fauteurs de désordre. Sinon, on prive le citoyen de ses droits légitimes. La protection souhaitée devient oppressive. Contre

62. A. Le Goff, La Neutralité religieuse de l'État..., p. 285.

63. CAA Nantes 4 février 1999, Association civique de Joué-Langueurs et autres, Rec. 498, DA, 1999, nº 189.

64. CE 6 juin 1947, Union catholique des hommes du diocèse de Versailles, Rec. 250.

65. CE 21 mars 1990, Commune de la Roque d'Anthéron, req. $\mathrm{n}^{\circ} 76.765$.

66. G. Le Bras, «Le Conseil d’État régulateur de la vie paroissiale», EDCE, 1950, p. 63-76.

67. M. Hauriou, Précis de droit administratif et de droit public, p. 637.

68. CE 19 mars 1909, Deguille, Rec. 311, D., 1910. III. 121 (concl. Saint-Paul).

69. $\mathrm{CE}_{1}{ }^{\mathrm{er}}$ mai 1914, Abbé Didier, Rec. 515.

70. Un siècle de laïcité, EDCE, $\mathrm{n}^{\circ}$ 55, 2004, p. 309.

71. CE 11 mai 1938, Mémeteau, Rec. 408; CE 12 juillet 1938, Rattier, Rec. 661; CE 25 janvier 1939, Abbé Marzy, Rec. 709; CE 3 décembre 1954, Rastouil, Rec. 639, AJDA, 1955. II. bis p. 1 (chron. M. Long). 
l'oppression, la conscience individuelle réclame le droit de résistance ${ }^{72}$.

«Ce caractère traditionnel demeure même si la procession n'a pas eu lieu depuis plusieurs années en raison d'une mesure administrative d'interdiction illégale ${ }^{73}$.

Elle est devenue, de surcroît, plus exigeante à l'égard des mesures de police interdisant, même pour la prévention à des atteintes éventuelles à l'ordre public, des processions non traditionnelles ${ }^{74}$.

En effet, pour ces autres manifestations religieuses, elles doivent être autorisées au cas par cas, sous le contrôle vigilant du juge administratif. Ce dernier examine la mesure de police d'interdiction au regard du trouble à l'ordre public susceptible d'être prévenu par un tel acte.

D'ailleurs, depuis 1920, toutes les interdictions trop générales et absolues sont annulées pour des motifs concentrés en deux formules ${ }^{75}$, que le Conseil d'État emploie indifféremment.

C'est pourquoi est annulé l'arrêt préfectoral interdisant une messe en plein air « dès lors que ladite cérémonie n'était pas de nature à menacer la tranquillité ou la sécurité publique dans des conditions telles qu'il ne put être paré à tout danger par des mesures de police appropriées ${ }^{76}$.

\section{Une limite importante:}

\section{la liberté religieuse des usagers}

Si la laïcité suppose de l'État et de ses agents une attitude non discriminatoire sur le plan religieux (une attitude voire une obligation d'abstention), elle lui impose aussi une attitude de préservation de la liberté de conscience des autres usagers (croyants ou non) en endiguant toute attitude religieuse de prosélytisme, perturbatrice, revendicative voire agressive, dans la sphère publique (obligation d'action), de la part de certains usagers.

La neutralité religieuse des lieux publics doit donc être conciliée avec la liberté religieuse des usagers.

«Nul doute que le régime juridique est bien différent entre les agents employés dans un service public et les usagers de ce même service. En effet, il ne peut y avoir de nombreuses restrictions à la liberté religieuse de l'usager. On ne peut pas raisonnablement envisager qu'une personne portant un foulard islamique soit obligée de le retirer en allant déposer un colis à la poste ${ }^{77}$.

Toutefois, certains services publics ont admis dans le passé la soumission de leurs usagers à une réserve compatible avec la discipline du service ${ }^{78}$.

Mais, ce n'est véritablement que dans le cadre du service public de l'Éducation nationale que la neutralité religieuse est imposée avec force aux usagers, les enseignés, avec la même fermeté avec laquelle elle s'applique aux enseignants.

C'est ainsi que, pour clore un contentieux administratif ${ }^{79}$ abondant relatif au port $\mathrm{du}$ " foulard islamique » dans les écoles publiques par des jeunes filles de confession musulmane, a été votée la loi $\mathrm{n}^{\mathrm{0}}$ 2004-228 du 15 mars 2004 encadrant, en application du principe de laïcité, le port de signes ou de tenues manifestant une appartenance religieuse dans les écoles, collèges et lycées publics.

72. A. Fossier, Les Manifestations cultuelles..., p. 90.

73. Un siècle de laïcité, EDCE, $\mathrm{n}^{\circ}$ 55, 2004, p. 309. Voir aussi : CE 14 février 1936, Abbé Chapalain, Rec. 205; CE 12 février 1947, Abbé Delusseau, Rec. 471; CE 26 avril 1950, Abbé Dalque, Rec. 234.

74. CE 21 janvier 1966, Legastellois, Rec. 45, JCP, 1966. II. nº 14.568 (concl. Y. Galmot), AJDA, 1966, p. 435 (note J. Moreau).

75. Ces formules sont : - soit : «Considérant que l'arrêté a par la généralité de ses termes, prohibé toutes les manifestations extérieures du culte, ce qui comprendrait le port du viatique, les convois funèbres, et les cérémonies fondées sur les traditions locales... " (CE 5 août 1921, Boulze, Rec. 819; voir aussi CE 25 novembre 1921, Charpentier, Rec. 975; CE 7 avril 1922, Dacheux, Rec. 337 ; CE 11 août 1922, Dhaussy, Rec. 745; CE 9 février 1923, Lavalette, Rec. 136; CE 21 novembre 1923, Laurent, Rec. 739; CE 26 décembre 1923, Chanoine Auffret, Rec. 883; CE 14 mai 1924, Curé de Villefort, Rec. 465 ; CE 4 juillet 1924, Guerle, Rec. 640); - soit : «Considérant que l'arrêté municipal interdit toutes les processions qu'aucun motif tiré de la nécessité de maintenir l'ordre sur la voie publique, ne pouvant être invoqué pour légitimer la prohibition de cérémonies telles que celles qui ont pour objet le culte des morts, et celles qui sont consacrées par les habitudes et les traditions locales » (CE 17 novembre 1922, Giraud, Rec. 835; voir aussi: CE 9 mars 1923, Rousset, Rec. 227; CE 23 mars 1923, Giraud, Rec. 279; CE 6 juillet 1923, Thomas, Rec. 549; CE 28 novembre 1923, Deruy, Rec. 767; CE 9 juillet 1924, Lhommet, Rec. 654 ; CE 20 mars 1925, Pouzineau, Rec. 288).

76. CE 5 mars 1948, Jeunesse indépendante chrétienne féminine, Rec. 121 ; en sens contraire: CE 2 juillet 1947, Guillet, Rec. 293. Cette jurisprudence, par sa formulation, rappelle naturellement l'arrêt de principe, Benjamin (CE 19 mai 1933, Rec. 541, D., 1933. III. 354 (concl. Michel), S., 1934. III. 1 (concl. ; note A. Mestre), GAJA, $\mathrm{n}^{\circ}$ 52, Lachaume p. 324).

77. D. Dutrieux et F. Leclercq, Opérations funéraires et laïcité, Paris, Weka (Les experts du funéraire), 2004, p. 20.

78. L'obligation de réserve - notion en principe évoquée pour les seuls agents publics - signifie, lorsqu'elle s'impose à l'usager d'un service public, que celui-ci doit exprimer, en principe, dans les lieux d'exécution d'un tel service, ses sentiments, ses opinions voire ses passions avec une retenue imposée par les nécessités du fonctionnement du service (CE 23 mai 1906, dame Chauvin, S., 1908. III. 17 (note M. Hauriou) : admission de l'obligation de réserve pour les usagers du téléphone; CE 5 juin 1946, Delert, S., 1947. III. 72 : admission de l'obligation de réserve pour les lycéens).

79. Pour quelques exemples: CE Ass. 22 novembre 1992, Kherouaa et autres, Rec. 389, JCP, 1993. II. $\mathrm{n}^{\circ} 21.998$ (note P. Tedeschi), RDP, 1993, p. 220 (note P. Sabourin), D., 1993, p. 108 (note G. Koubi), D., 1993. IR. 4, LPA, 24 mai 1993, nº 62, p. 4 (note G. Lebreton), GP, 1993, 2, p. 8 (note D. Mardesson), RFDA, 1993, p. 112 (concl. D. Kessler), AJDA, 1992, p. 788 et 790 (ch. C. Maugüé et R. Schwartz); CE 14 mars 1994, M ${ }^{\text {lle }}$ Yilmaz, Rec. 129, LPA, $1^{\text {er }}$ mars 1995 (n ${ }^{\circ}$ 26), p. 23 (note J.-F. Flauss), RDP, 1996, p. 221 (note A. de Lajartre); CE 10 mars 1995, M. et M Me Aoukili, Rec. 122, EDCE, 1995 (nº 47), p. 329, AJDA, 1995, p. 332 (concl. Y. Aguila), JCP, 1995. II. n 22431 (note N. Van Tuong), JCP, 1995. IV. n 243, RFDA, 1995, p. 631 (n ${ }^{\circ} 12$ ), D., 1995, p. 365 (note G. Koubi); CE 20 mai 1996, M Mle Mabchour, RFDA, 1997, p. 162 ; CE Sect. 20 mai 1996, Ministre de l'Éducation nationale c. Ali, Rec. 187, AJDA, 1996, p. 709 (note G. Koubi) ; CE 20 mai 1996, Ministre de l'Éducation nationale c. Khalid et $M^{\text {me }}$ Stefiani, Rec. 460 ; CE 26 juillet 1996, Université de Lille II, req. ${ }^{\circ} 170.106$; CE 9 octobre 1996, Ministre de l'Éducation nationale c. UNAL, D., 1996, p. 247 ; CE 27 novembre 1996, Ligue islamique du Nord, Rec. 461, JCP, 1997. II. nº 22808 (note B. Seiller), DA, 1997, $\mathrm{n}^{\circ} 6$ (note R. S.), LPA, 10 mars 1997 (n 30 ), p. 10 (note N. Van Tuong), EDCE, 1996 (n 48), p. 132, RFDA, 1997, p. 171; CE 27 novembre 1996, Ministre de l'Éducation nationale c. Mlle Akbaba, RFDA, 1997, p. 171; CE 27 novembre 1996, Ministre de l'Éducation nationale c. Époux Naderan, RFDA, 1997, p. 171; CE 27 novembre 1996, Époux Wissadane et époux Chedouane, Rec. 462; CE 20 octobre 1999, Ministre de l'Éducation nationale c. Aït Ahmad, Rec. 776, JCP, 2000. II. n 10.306 (note G. Koubi et G.-J. Guglielmi), D., 2000, p. 251 (concl. R. Schwartz), RTDH, 2001, p. 77 (concl. ; note Z. Anseur). 
En application de cette loi, et au regard du nouvel article L 141-5-1 du Code de l'éducation, issu de ce texte législatif:

«Dans les écoles, les collèges et les lycées publics, le port de signes ou tenues par lesquels les élèves manifestent ostensiblement une appartenance religieuse est interdit.

Le règlement intérieur rappelle que la mise en œuvre d'une procédure disciplinaire est précédée d'un dialogue avec l'élève ».

C'est pourquoi le port par un élève de confession sikhe d'un sous-turban est considéré comme un signe manifestant ostensiblement une appartenance religieuse et peut donc légalement être interdit ${ }^{80}:$ « [...] dès lors qu'en persistant à porter le sous-turban ou keski sikh, il adoptait une tenue le faisant reconnaître immédiatement comme appartenant à la religion sikhe, et cela sans que l'administration n'ait à s'interroger sur la volonté de l'intéressé d'adopter une attitude de revendication de sa croyance ou de prosélytisme, ni à établir que l'attitude du requérant était de nature à troubler l'ordre public ${ }^{81}$.

Par analogie, est légale la décision administrative interdisant le port du voile sur les photographies d'identité, présentées à l'appui d'une demande de carte d'identité nationale; cette décision n'est pas considérée comme attentatoire à la liberté religieuse ${ }^{82}$. D'ailleurs, dans de nombreux États européens, «[...] la seule règle à respecter [est] que les traits du visage ainsi que la couleur des yeux et des cheveux apparaissent clairement sur les documents d'identité ${ }^{83}$.

In fine, les autorisations d'absence d'élèves pour l'exercice d'un culte ne sont possibles que si les absences sont compatibles avec les obligations scolaires et donc l'ordre public de l'établissement; dès lors, les refus de telles autorisations ne sont légaux que s'ils sont justifiés par de telles contraintes intérieures à l'établissement scolaire ${ }^{84}$.

Mais, «La loi [du 15 mars 2004] ne concerne pas les parents d'élèves. Elle ne s'applique pas non plus aux candidats qui viennent passer les épreuves d'un examen ou de concours dans les locaux d'un établissement public d'enseignement et qui ne deviennent pas de ce seul fait des élèves de l'enseignement public. Ceux-ci doivent toutefois se soumettre aux règles de respect de l'ordre et de la sécurité, à permettre la vérification de l'identité des candidats ou à prévenir les risques de fraudes ${ }^{85}$.

«Cette différence [de traitement juridique entre l'usager du service public et son agent au regard de la neutralité religieuse] est justifiée par la différence de nature entre la situation de l'usager et celle de l'agent public. Personnels et usagers ne sont, en effet, pas dans la même relation ${ }^{86}$.

De plus, et en principe, l'usager ne fait que passer, il n'a pas en principe le rôle que l'agent public y a, au-delà de sa "permanence » professionnelle. Dès lors, l'attitude de l'agent public, particulièrement sur le plan de l'expression de ses convictions, entre autres religieuses, au regard de l'exécution du service public auquel il participe, est très importante.

La neutralité de l'agent public a donc une incidence directe sur celle du service public, et par là même, sur le lieu où il exerce ses fonctions.

\section{B. Une condition importante : le devoir de neutralité des agents publics}

\section{Le contenu du devoir de neutralité}

L'agent public se doit d'être neutre dans l'exercice de ses fonctions.

En effet, «Le principe de laïcité, dont le principe de neutralité est un élément, fait obstacle à l'expression des convictions religieuses des personnels dans le cadre du service public. Cette interdiction est absolue $»{ }^{87}$.

Cela s'explique pour deux raisons essentielles.

D'une part, parce que l'agent public doit assurer, par son attitude, dans le cadre de l'exécution des services publics, et plus particulièrement dans la prestation qu'il fournit à l'usager, une égalité de traitement, et par là même il doit éviter d'afficher un quelconque comportement discriminatoire $^{88}$. Cet aspect de l'obligation de neutralité de l'agent public illustre avec évidence son lien avec le principe d'égalité dont elle est issue. Tout agent public doit assurer « un service public dans les mêmes conditions, de façon impartiale et sans discrimination de caractère politique, philosophique ou religieux entre les usagers et autres personnes ${ }^{89}$.

80. Par comparaison, il convient de souligner qu'au Royaume-Uni, la position est inverse de la solution française. En effet, les sikhs peuvent conserver leur turban parce que la Chambre des Lords a estimé que les obliger à l'enlever aurait constitué une « discrimination raciale indirecte ». Ce qui, par suite, a obligé le législateur britannique à adapter la loi anglaise au profit des sikhs concernant le port des casques de chantier ou de motocycliste (voir D. Fairgriewe, «Un État non laïc : le Royaume-Uni», p. 14-15).

81. TA Melun 19 avril 2005, M. Gurdial Singh, AJDA, n 17, 2005, p. 917 (obs. E. Royer).Le juge administratif relève, au titre de la «bonne volonté de l'administration ", la proposition qui a été faite à l'élève d'abandonner le sous-turban pour un filet de protection afin de protéger et de maintenir l'intégrité absolue de sa chevelure tout en respectant les règles d'hygiène et de sécurité.

82. CE 27 juillet 2001, Fonds de défense des musulmans en justice, req. ${ }^{\circ} 216.903$. Pour une autre application, voir CE 24 octobre 2003, $M^{m e}$ Fatima Benchemackt, req. $\mathrm{n}^{\circ} 250.084$ : refus légal de renouvellement de passeport du fait que la demande est accompagnée de photographies d’identité où la demanderesse y apparaît voilée.

83. C. Pauti, «L'affaire du crucifix dans les écoles italiennes », AJDA, $\mathrm{n}^{\circ}$ 14, 2004, p. 746

84. CE Ass. 14 avril 1995, Koen et Consistoire central des israélites de France, Rec. 169 (concl. Y. Aguila), D., 1995, p. 481 (note G. Koubi), RFDA, 1995, p. 585 (concl.), AJDA, 1995, p. 501 (concl. J.-F. Stahl et D. Chauvaux), RDP, 1997, p. 1783 (note S. Poulpiquet).

85. Circulaire du 18 mai 2004 relative à la mise en œuvre de la loi $n^{\circ}$ 2004-228 du 15 mars 2004 encadrant, en application du principe de laïcité, le port de signes ou de tenues manifestant une appartenance religieuse dans les écoles, collèges et lycées publics, JORF, 22 mai 2004, p. 9034.

86. M. Picard, «Le principe de laïcité et son application aux agents des services publics », CFP, nº 217, 2002, La Laïcité, p. 6.

87. Ibid.

88. O. Dord, note sous CE 22 novembre 2004, Ministre de la Jeunesse, de l'Éducation nationale et de la Recherche c. M. A...,AJDA, $\mathrm{n}^{\circ}$ 13, 2005, p. 736.

89. V. Kondylis, Le Principe de neutralité dans la fonction publique, p. 8. 
«Il en résulte pour les agents publics une obligation concrète ${ }^{90}$. Dans la mesure où le respect qu'ils doivent à leur devoir de neutralité, composante de leur devoir de réserve ${ }^{91}$, leur impose des limitations à leur liberté d'expression, cette dernière est limitée.

Sachant que leur obligation de réserve est plus ou moins étendue, selon leur rang, les circonstances dans lesquelles ils s'expriment et selon qu'ils exercent ou non un mandat syndical.

Dès lors, il apparaît avec évidence que, dans le cadre de leurs fonctions, et durant le temps de leur service, les agents publics n'ont pas le droit d'exercer leur religion, de la manifester de manière ostentatoire voire provocatrice. Et ce, que leur attitude soit volontaire ou non, dans la mesure où personne ne peut jamais savoir à l'avance comment un usager peut vivre et ressentir un signe religieux porté par un agent public ou une attitude religieuse d'un tel agent.

C'est pourquoi «[...] les maîtres de l'enseignement public sont tenus, dans l'exercice de leur profession, à une stricte “impartialité" à l'égard de la religion »" ${ }^{92}$. Ainsi, sont constitutives d'une faute personnelle, pouvant engager sa responsabilité personnelle, les paroles injurieuses et grossières sur Dieu, sur les religions et sur les ministres du culte, prononcées par un instituteur, dans le cadre de ses enseignements; en effet, ces propos sont blessants à l'égard des enfants, et incidemment, de leur famille si ces dernières sont sincèrement croyantes ${ }^{93}$.

D'autre part, l'agent public doit, dans le cadre de l'exercice de ses fonctions, et au nom de la neutralité du service public, et par suite en respectant son obligation de réserve, adopter une attitude, spécialement à l'égard de ses collègues, qui ne reflète pas ses opinions. Il doit, durant le temps de son service, extérioriser avec retenue ses conceptions politiques, religieuses, philosophiques, etc.

"Cette règle est si forte qu'elle s'applique même hors service à travers le devoir de réserve qui impose à l'agent de veiller à ce que son comportement ne retentisse pas sur le service. Sont ainsi proscrits les actes ou déclarations à la fois publics et critiques qui porteraient atteinte à la neutralité des services publics ${ }^{94}$.

À cet égard, le statut général des militaires est des plus explicite. En effet, et aux termes des deux premiers alinéas de l'article 4 de la loi n ${ }^{\circ}$ 2005-270 du 24 mars 2005, «Les opinions ou croyances, notamment philosophiques, religieuses ou politiques, sont libres.

Elles ne peuvent cependant être exprimées qu'en dehors du service et avec la réserve exigée par l'état militaire $[\ldots] »$.

C'est au regard des deux aspects du devoir de neutralité des agents publics que le juge administratif considère que «Si les agents du service de l'enseignement public bénéficient comme tous les autres agents publics de la liberté de conscience qui interdit toute discrimination dans l'accès aux fonctions comme dans le déroulement de la carrière qui serait fondée sur leur religion, le principe de laïcité fait obstacle à ce qu'ils disposent, dans le cadre du service public, du droit de manifester leurs croyances religieuses».

Et il ajoute qu' " il n'y a pas lieu d'établir une distinction entre les agents de ce service selon qu'ils sont ou non chargés des fonctions d'enseignement ${ }^{95}$.

En effet, « La conciliation des principes conduit [...] à la reconnaissance pour les agents de la liberté de croyance, mais aussi à la négation, dans le cadre du service, du droit d'exprimer et de manifester leur foi. [...] $»^{96}$.

In fine, il convient de souligner que le respect du principe de neutralité ${ }^{97} s^{\prime}$ impose aussi à l'administration relativement à l'accès à la fonction publique.

C'est ainsi que le juge administratif condamne les discriminations fondées sur les opinions religieuses des candidats à l'entrée ou au maintien dans la fonction publique $^{98}$.

\section{L'intérêt de la neutralité de l'agent public pour celle du lieu public}

Dans l'absolu, un lieu est neutre; il peut par des ornements, des affiches, devenir " engagé », mais derrière toute apposition de cette nature, se trouve obligatoirement la main humaine, celle le plus souvent d'un agent public.

Dès lors, un lieu n'est pas seulement neutre en luimême mais il l'est aussi par la manière dont il est aménagé et dont il est utilisé.

Un lieu public est facilement neutre et peut tout aussi facilement ne plus l'être, et par là même, être instrumentalisé au profit d'une cause quelconque, en fonction de l'aménagement particulier dont il peut faire l'objet par les

90. M. Picard, «Le principe de laïcité... », p. 5.

91. «La marque de cette neutralité c'est l'obligation de réserve. Cette obligation n'est qu'un corollaire du principe d'égalité de tous devant le service public qui doit fonctionner sans discrimination ni faveur » (J.-M. Lavieille, "Les principes fondamentaux de l'enseignement dans le droit positif français », AJDA, 1978, p. 192).

92. R. Charvin et J.-J. Sueur, Droits de l'homme et libertés de la personne, $4^{\mathrm{e}}$ éd., Paris, Litec, 2002, p. 163, nº 120.

93. TC 2 juin 1908, Girodet c. Morisot, Rec. 597 (concl. Tardieu), S., 1908, III. 81 (concl.; note M. Hauriou), DP, 1908. III. 57.

94. L. Wauquiez-Motte, «Laïcité et neutralité de l'État », p. 7.

95. CE 3 mai 200o, Avis n ${ }^{\circ} 217017, M^{\text {lle }}$ Marteaux, Rec. 169, AJDA, 2000, p. 602 (ch. M. Guyomar et P. Collin), RFDA, 2000, p. 899, DA, 2000, nº 189 (note R. S.), RFDA, 2001, p. 146 (concl. R. Schwartz), D., 2000, p. 747 (note G. Koubi), RRJ, 2001-4 (II), p. 2107 (note G. Armand).

96. M. Guyomar et P. Collin, Chronique sous CE 3 mai 2000, Avis n $217017, M^{\text {lle }}$ Marteaux, AJDA, 2000, p. 604.

97. E. Aubin, Droit de la fonction publique, p. 94-100; J.-M. Auby, J.-B. Auby, D. Jean-Pierre et A. Taillefait, Droit de la fonction publique, p. $135-137$ ( ${ }^{\circ}$ 239$240)$; T. Ben Salah, Droit de la fonction publique, p. 107-114 (nº 139-149); J.-F. Lachaume, La Fonction publique, p. 45-51 et p. 87-9o. Il en va de même dans le secteur privé, au regard entre autres de l'article L 122-45 du Code du travail, dans la mesure où « les convictions religieuses, sauf clause expresse, n'entrent pas dans le cadre du contrat de travail» (Un siècle de laïcité, EDCE, n ${ }^{\circ} 55,2004$, p. 343).

98. CE 28 avril 1938, D $D^{\text {lle }}$ Weiss, D., 1939. III. 41 (note M. Waline; concl. Dayras), RDP, 1938, p. 553 ; CE Sect. 25 juillet 1939, $D^{l l e}$ Beis, Rec. 524 ; CE 8 décembre 1948, Demoiselle Pasteau, Rec. 464; CE 3 mai 1950, D ${ }^{\text {lle }}$ Jamet, Rec. 247 ; CE 7 juillet 1954, Janinet, AJDA, 1954, p. 398. 
seuls agents publics qui y exercent le service public qui leur a été confié.

Ainsi, en est-il lorsque les agents publics tolèrent la distribution ou distribuent eux-mêmes des tracts exprimant des convictions religieuses ${ }^{99}$ ou politiques ${ }^{100}$ voire organisent une information qui pourrait être considérée comme attentatoire aux convictions politiques ${ }^{101}$ ou religieuses ${ }^{102}$ des usagers ou de leurs parents.

Et ce, parce qu'alors, l'agent instrumentalise le service public, et incidemment le lieu d'exécution de celui-ci, afin de faire de ce dernier un lieu de propagande ; ce qui à coup sûr amène l'agent à violer son devoir de neutralité ${ }^{103}$.

Ce qui explique que ces attitudes, violant la neutralité du service public, justifient l'engagement de la responsabilité disciplinaire de l'agent public, sous le contrôle du juge administratif, qui appréciera la sanction en fonction de la «nature et du caractère ostentatoire » du signe porté par, ou de l'action incriminée de, l'agent.

En effet, le fait pour un agent public de manifester dans l'exercice de ses fonctions, cette fois-ci, ses croyances religieuses, notamment en portant un signe destiné à marquer son appartenance à une religion, constitue un manquement à ses obligations et justifie son licenciement ${ }^{104}$ ou - ce qui revient au même - le non-renouvellement de son contrat ${ }^{105}$.

Dès lors, le port ostentatoire d'un signe religieux, tel que le foulard islamique, et le refus de le retirer malgré l'ordre reçu de la hiérarchie constitue une faute disciplinaire pour un fonctionnaire investi de prérogatives de puissance publique ou investi de fonctions de représentations ${ }^{106}$.

Ce que confirme d'ailleurs la Cour européenne des droits de l'homme ${ }^{107}$ qui a admis comme compatible avec la liberté de religion, consacrée à l'article 9 de la Convention européenne de sauvegarde des droits de l'homme et des libertés fondamentales, l'interdiction faite à une enseignante de porter le foulard islamique à l'école, en s'appuyant sur le fait qu'étaient en cause des enfants en bas âge
( «vu surtout le bas âge des enfants » dit l'arrêt; de même, plus indirectement, ajoute-t-il qu' " il est bien difficile d'apprécier l'impact qu'un signe extérieur fort tel que le port du foulard peut avoir sur la liberté de conscience et de religion d'enfants en bas âge »), supposés être impressionnables et influençables sur la question de la religion.

Toutefois, cette décision laisse ouverte le port du foulard par des membres de la communauté éducative, enseignants ou non, dans des établissements scolaires où l'on peut considérer que les enseignés sont «moins impressionnables et influençables ». Par suite, cela serait admissible dans les lycées voire dans les collèges. De plus, on peut penser que la jurisprudence de la Cour européenne ne va pas estimer que les usagers des services publics, souvent ou du moins majoritairement adultes ou en voie de l'être, puissent être impressionnés ou influencés par de tels signes religieux. En somme, la jurisprudence évoquée semble plus libérale que la jurisprudence française par ses silences.

En effet, si la jurisprudence européenne admettait cette interprétation, cela remettrait en cause la jurisprudence administrative française concernant une sorte d'interdiction générale et absolue du port du voile islamique par les agents de tous les services publics.

C'est pourquoi, il n'est pas inintéressant de faire le parallèle avec la situation juridique des enseignants des établissements confessionnels sous contrat (simple ou d'association).

La consécration, par la loi Debré du 31 décembre 1959, du caractère propre de tels établissements privés, malgré la neutralité du service public auquel ils sont associés, leur permet - c'est-à-dire tant à leur personnel de direction qu'à leurs enseignants - de défendre leurs valeurs morales voire religieuses à l'occasion de leur action d'administration de l'établissement ou pédagogique.

Ainsi, l'enseignant privé - bien que parfois principalement payé par l'État et dont le statut a récemment grandement évolué au profit d'une forte intégration dans la

99. TA Lille $1^{\text {er }}$ juillet 2003, Schneerberger, AJDA, $\mathrm{n}^{\circ} 8,2004, \mathrm{p} .461$ : n'est pourtant pas considérée comme contraire au principe de neutralité la distribution de revues religieuses aux détenus, même si ces revues émanent d'une congrégation reconnue sectaire par une commission d'enquête parlementaire.

100. TA Nice 30 mars 1984, Caressa, Rec. 656

101. CE 8 novembre 1985, Ministre de l'Éducation nationale c. Rudent, Rec. 316, RDP, 1987, p. 244 (note F. Llorens), AJDA, 1985 , p. 712 (ch. S. Hubac et M. Azibert), D., 1986. IR. 48, RDP, 1986, p. 244 (note F. Llorens), RFDA, 1986, p. 630 (concl. M. Laroque); CE $1^{\text {er }}$ mars 1993, Ministre de l'Éducation nationale c. Association des parents d'élèves de l'enseignement public de Montpellier, AJDA, 1993, p. 881 (note B. Toulemonde), Quot. Jur., 22 juin 1993, $\mathrm{n}^{\circ}$ 49, p. 5 ; CE 6 novembre 1991, Ministre de l'Éducation nationale c. Confédération des groupes autonomes de l'enseignement public, Rec. 377, LPA, 30 juillet $1993, \mathrm{n}^{\circ} 91, \mathrm{p} .35$ (note A. Thiriot), $D A, 1991, \mathrm{n}^{\circ} 521$.

102. CE 6 octobre 2000, Association Promouvoir et autres, Rec. 391, AJDA, 2000, p. 609 (ch. M. Guyomar et P. Collin), AJDA, 200o, p. 1060 (concl. S. Boisard), RFDA, 2000, p. 1311 (obs. J. Morange), LPA, 15 décembre 2000 (n ${ }^{\circ} 250$ ), p. 9 (note O. Lecucq), DA, 2000, $\mathrm{n}^{\circ} 192, D ., 2001, \mathrm{p} .590$ (note E. Boitard), JCP, 2000. II. p. 2006. Voir, dans le même sens: CEDH 7 décembre 1976, Kjeldsen et autres, AFDI, 1977, p. 489 (ch. R. Pelloux), CDE, 1978, p. 359 (ch. G. Cohen-Jonathan), JDI, 1978, p. 702 (ch. P. Rolland), in F. Sudre, J.-P. Marquénaud, J. Andriantsimbazovina, A. Gouttenoire et M. Levinet, Les Grands arrêts de la Cour européenne des droits de l'homme, 2 éd., Paris, PUF (Thémis, Les grandes décisions de la jurisprudence), 2004, p. 441 (obs. M. Levinet).

103. G. Koubi, «À la recherche d'une définition spécifique de l'obligation de neutralité des fonctionnaires et agents publics», $L P A, 26$ juin $1991, \mathrm{n}^{\circ} 76$, p. 21.

104. CE 3 mai 2000, Avis $\mathrm{n}^{\circ}$ 217017, $M^{\text {lle }}$ Marteaux, préc.

105. TA Paris 17 octobre 2002, $M^{m e} E \ldots, J C P A, 2002, \mathrm{n}^{\circ} 1150$ (note D. Jean-Pierre).

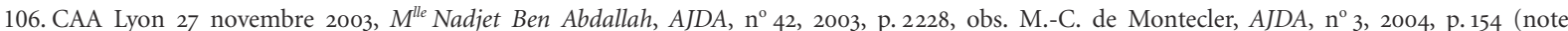
F. Melleray), AJFP, $\mathrm{n}^{\circ}$ 2, 2004, p. 88 (obs. J. Mekhantar et note F. Lemaire). Voir aussi: TA Lyon 8 juillet 2003, M ${ }^{\text {lle }}$ Nadjet Ben Abdallah, AJDA, ${ }^{\circ} 36$, 2003, p. 1951; TA Versailles 14 mai 1992, $D^{l l e}$ Brazza, Savoir, 1992, p. 549, RT, 1995, nº 10, p. 646. Voir, par analogie : CA Saint-Denis-de-la-Réunion 9 septembre 1997, D., 1998, p. 546 (note S. Farnocchia); CA Paris 16 mars 2001, Habiba Charni, RJS, 11/2001, $\mathrm{n}^{\circ} 1252$.

107. CEDH 15 février 2001, Dahlab c. Suisse, AJDA, 2001, p. 482 (note J.-F. Flauss). 
fonction publique d'État ${ }^{108}$ - peut et même doit avoir une attitude pédagogique visant à valoriser la spécificité éducative de son établissement c'est-à-dire, le cas échéant, les valeurs confessionnelles de ce dernier, tout en devant éviter de porter atteinte à la liberté de conscience des enseignés et respecter la neutralité des enseignements (au regard du respect dû aux programmes nationaux d'enseignement).

Malgré tout, à l'obligation de neutralité des enseignants publics, à leur abstention d'exprimer leurs convictions profondes, particulièrement d'ordre religieux, répond la possibilité d'exprimer son adhésion aux valeurs de l'établissement d'enseignement privé pour tout enseignant y exerçant ${ }^{109}$.

Dès lors, autant l'extériorisation de leurs croyances religieuses est fermement interdite aux agents publics, spécifiquement aux enseignants, autant elle est reconnue à ceux du privé, particulièrement si elle est en rapport étroit avec le «caractère propre » de l'établissement dans lequel ils enseignent.

Ce qui rend toutefois leur tâche aussi malaisée d'exercice qu'ambiguë en termes de définition.

Puisque, tout en pouvant affirmer leur identité religieuse, ils se doivent malgré tout de ne pas violer la conscience des élèves. Ils peuvent montrer mais ne pas choquer; toutefois, montrer peut suffire à choquer.

Mais, n'oublions pas que si ces élèves sont dans des établissements confessionnels, c'est parce que leurs parents l'ont voulu.

Il est, par ailleurs, refusé à un gardien d'office HLM de s'absenter pendant ses heures de service pour prier; comme le reconnaît le juge administratif, si « la liberté de culte présente le caractère d'une liberté fondamentale, [elle est, en l'espèce, incompatible] avec les nécessités du fonctionnement normal du service ${ }^{110}$.

En effet, aucun texte n'impose la prise en compte de toutes les fêtes religieuses pour organiser l'activité administrative; mais, les chefs de service peuvent, sous le contrôle du juge, et en fonction des nécessités du service et donc de son fonctionnement normal, accorder aux agents publics des autorisations d'absence pour participer à une fête religieuse correspondant à leur confession, et ce, depuis des circulaires du 7 avril 1964 et du 23 septembre 1967.

Mais, il s'agit d'une faculté et non d'une obligation pour le chef de service: il n'est pas tenu d'accorder une telle absence si le fonctionnement normal du service l'empêche; en revanche, un refus, en l'absence de remise en cause du fonctionnement du service et alors que l'absence était compatible avec ce dernier, est considéré comme illégal, car abusif, par le juge administratif ${ }^{111}$.
Enfin, le juge administratif valide la sanction prononcée contre un agent public ayant utilisé les moyens de communication du service au profit d'un mouvement religieux et étant apparu sur le site de ce mouvement en qualité de membre de celui-ci avec la mention de son mail professionnel. De tels agissements constituent « [...] un manquement au principe de laïcité et à l'obligation de neutralité qui s'impose à tout agent public ${ }^{112}$.

Toutefois, l'employeur public ne peut pas s'octroyer le droit de contrôler tous les écrits et les ouvrages, avant publication, de l'agent public, et ce, même si ceux-ci concernent les fonctions qu'il exerce et critiquent l'action du gouvernement auquel ses fonctions l'associent ${ }^{113}$.

En principe, la neutralité religieuse des lieux publics constitue une obligation juridique, liée à l'application du principe de laïcité, et ce, de manière apparemment générale et absolue.

Cette obligation est d'autant plus générale qu'elle concerne tant les locaux, modalité d'organisation du service public, que les agents publics qui l'exécutent.

En effet, un lieu n'est pas simplement neutre en soi, il l'est au regard de la façon dont il est aménagé, décoré, ou encore, utilisé.

Toutefois, est-ce que cette obligation s'impose aux lieux publics de manière véritablement absolue?

Rien n'est moins sûr.

En effet, il existe certains lieux publics qui, par essence, sont affectés aux cultes, dont la destination est exclusivement cultuelle.

Par suite, pour ces lieux, que sont les cimetières, les édifices cultuels ou les aumôneries, il est impossible de leur appliquer strictement une quelconque obligation de neutralité religieuse au risque de nier - pour plagier la loi Debré de 1959 - leur « caractère propre », leur spécificité, en un mot, leur raison d'être.

Dès lors, l'obligation juridique de neutralité, au regard de ces quelques lieux, ne peut s'appliquer que de manière, parfois, partielle ou ne peut nullement s'appliquer.

\section{La neutralité religieuse dans les lieux publics : une application nuancée}

La rigueur du principe de neutralité religieuse des lieux publics est parfois relativisée au regard des situations pratiques et des exemples concrets de gestion de tels lieux.

Ainsi en est-il pour les cimetières, normalement aconfessionnels, mais finalement parfois moins neutres, et par

108. Loi n 2005-5 du 6 janvier 2005 relative à la situation des maîtres des établissements d'enseignement privé sous contrat.Voir B. Toulemonde, «Les maîtres des établissements d'enseignement privés entre droit public et droit privé : une hybridation législative ", AJDA, $\mathrm{n}^{\circ}$ 9, $2005, \mathrm{p} .478-485$.

109. G. Marcou, «La liberté de l'enseignement et la liberté des enseignants », RDP, 1980, p. 35-82.

110. CE 16 février 2004, M. Ahmed B..., AJDA, $\mathrm{n}^{\circ}$ 8, 2004, p. 416, obs. S. Brondel, AJDA, ${ }^{\circ}$ 15, 2004, p. 822 (note G.-J. Guglielmi et G. Koubi), JCP A,

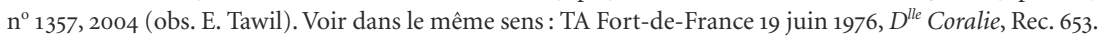

111. CE 12 février 1997, Henny, DA, 1997, nº 248 (note C. M.), LPA, 12 janvier 1998 ( ${ }^{\circ}$ 5), p. 8 (note F. Bouscau). Les solutions sont comparables en droit privé: Un siècle de laïcité, $E D C E, \mathrm{n}^{\circ} 55,2004, \mathrm{p} .344-345$.

112. CE Sect. 15 octobre 2003, M. O.., AJDA, 2003, p. 1959 (obs. Z. Ait-El-Kadi), AJFP, nº 1, 2004, p. 31 (obs. O. Guillaumont).

113. CE 29 décembre 2000, Syndicat Sud Travail et autres, D. Soc., 2001, p. 263 (concl. P. Fombeur). La Cour de justice des communautés européennes considère, quant à elle, qu'une telle exigence est légitime (CJCE, 6 mars 2001, Connoly, D., 2002. SC. 690 (obs. J. Rideau)). 
là même, plus favorables à l'exercice de la liberté religieuse que d'autres lieux publics.

Et ce, d'autant plus que face à la mort, les principes et les règles s'effacent souvent au profit du respect dû aux défunts et à leurs dernières volontés (A).

De surcroît, dans des lieux encore plus symboliques que sont les aumôneries et les lieux cultuels, bien que ces lieux soient publics (pour les deux premiers), il est difficile voire impossible d'imposer le respect de la neutralité religieuse.

En effet, ces lieux sont, par principe, religieux, voire au service d'une religion. Par suite, leur appliquer le principe de neutralité religieuse serait un non-sens voire, plus exactement, un contresens au regard de leur affectation, de leur raison d'être.

Dès lors, pour ces lieux, pourtant publics, la neutralité religieuse y est niée au profit, en toute logique, de l'expression de la liberté religieuse (B).

\section{A. Un principe ébranlé : le lieu funéraire ${ }^{114}$}

\section{La neutralité religieuse des cimetières}

Depuis les lois du 14 novembre 1881 et du 5 avril 1884 , il est interdit dans les cimetières communaux d'établir une séparation à raison de la différence des cultes. En effet, cette loi a abrogé l'article 15 du décret du 23 prairial, an XII qui prévoyait l'obligation pour les communes de réserver dans les cimetières une surface proportionnelle aux effectifs de fidèles des différents cultes ${ }^{115}$ et faisait aux familles obligation de déclarer le culte du défunt ${ }^{116}$.

De plus, l'article 28 de la loi du 9 décembre 1905 sur la séparation des Églises et de l'État dispose qu' «il est interdit à l'avenir d'élever ou d'apposer aucun signe ou emblème religieux sur les monuments publics ou en quelque emplacement public que ce soit, à l'exception des édifices servant au culte, des terrains de sépulture dans les cimetières, des monuments funéraires, ainsi que des musées et expositions».

Cet article emporte deux conséquences distinctes mais quelque peu contradictoires.

En premier lieu, toutes les parties publiques du cimetière, telles que les espaces et allées, sont soumises à cette neutralité religieuse, et par suite, ne peuvent recevoir d'emblèmes religieux sauf s'ils existaient avant l'entrée en vigueur de la loi de 1905.

Ainsi, les parties publiques du cimetière doivent strictement respecter la laïcité du lieu. Par suite, le maire doit certes respecter les cultes religieux des divers utilisateurs du lieu mais doit, en même temps, veiller à ce que ce dernier reste neutre. Il doit donc veiller, par exemple, à ce que les cultes n'organisent pas dans l'enceinte des cimetières des manifestations cultuelles en dehors des inhumations.

Le Conseil d'État l'exprime clairement et fermement dans sa jurisprudence ancienne et constante sur ce point ${ }^{117}$.

Toutefois, et en second lieu, les tombes et monuments funéraires, étant de nature privée, bien que se trouvant dans un lieu public, peuvent être réalisés par les familles en étant ornés de signes ou emblèmes religieux. Le maire ne peut pas s'y opposer sans commettre une grave illégalité ${ }^{118}$.

Ainsi, les expressions religieuses ne peuvent pas vraiment être prohibées dans un cimetière.

Bien qu'aux termes de l'article R. 2223-8 du Code général des collectivités territoriales, «aucune opération autorisée par le maire consistant à inscrire le nom d'un défunt, ses dates de naissance et de mort, ainsi qu'éventuellement une épitaphe sur la construction présente sur une concession funéraire ne peut être placée sur les pierres tumulaires ou monuments funéraires sans avoir été préalablement soumise à l'approbation du maire », ce dernier ne pourrait en aucune façon interdire une inscription sous le prétexte qu'elle présente un caractère religieux de nature à porter atteinte à la liberté de conscience.

En effet, si le maire peut prendre des dispositions dans l'intérêt de l'hygiène et de la salubrité ${ }^{119}$, ou autoriser l'établissement d'un dépôt de vente d'un horticulteur à l'intérieur du cimetière ${ }^{120}$, il ne peut interdire les visites processionnelles (constituant une tradition se rattachant au culte des morts) d'un prêtre le dimanche des Rameaux et le jour de la Toussaint ${ }^{121}$, ni évidemment soumettre à autorisation préalable le dépôt au pied d'un monument aux morts présent dans le cimetière de gerbes ou objets contenant des inscriptions ou attributs religieux. Et ce, parce que le juge administratif considère qu'un tel acte porte atteinte aux droits des familles « de rendre à ceux des leurs, morts pour la France, un hommage de forme aussi normalement et traditionnellement respectable ${ }^{122}$.

De même qu'il ne lui appartient pas, en vertu de ses pouvoirs de police, de déterminer les dimensions et la hauteur des monuments funéraires édifiés dans les limites des concessions funéraires; et ce, même si la construction religieuse envisagée est conséquente ${ }^{123}$.

\section{La survivance voire la renaissance des cimetières et carrés confessionnels}

«La question des carrés ou regroupements confessionnels dans les cimetières montre une fois de plus qu'il

114. S. Guérard, «Les lieux d'inhumations et les sépultures », in Guide juridique de droit funéraire, D. Dutrieux (dir.), Paris, Weka, 2005.

115. David, Conclusions sur CE 11 juin 1875, Hallé et autres, Rec. 561; L. Aucoc, Conclusions sur CE 8 février 1868, Jousseaume, Rec., p. 139-140. Sous

l'empire du décret de l'an XII : « [...] chaque culte doit avoir un lieu d'inhumation particulier» (CE 8 février 1868, Jousseaume, Rec. 137).

116. CE 17 juillet 1861, Karatsch, Rec. 604; CE 13 mars 1872, Tamelier, Rec. 153.

117. CE 16 décembre 1913, Abbé Deguille, Rec. 307 (concl. Saint-Paul).

118. CE Sect. 18 février 1972, Chambre syndicale des entreprises artisanales du bâtiment de la Haute-Garonne, JCP, 1973. II. $\mathrm{n}^{\circ}$ 17.446 (obs. F. Bouyssou).

119. CE 23 décembre 1921, Auvray-Rocher, Rec. 1092.

120. CE 20 février 1935, Degroote, Rec. 216.

121. CE 8 avril 1911, Abbé Anselme, Rec. 164.

122. CE 23 décembre 1927, D $D^{l l e}$ Lucien, Rec. 257.

123. CE 21 janvier 1910, Gonot, D., 1911. III. 135 (concl. Saint-Paul). 
y a, parfois s'agissant du principe de neutralité, un écart conséquent entre la théorie et la pratique ${ }^{124}$.

Mais, il convient de ne pas oublier qu'un grand nombre des cimetières et carrés confessionnels préexistaient aux lois de 1881 et de 1884 .

\section{a. Les cimetières confessionnels}

En effet, avant l'abrogation de l'article 15 précité du décret de l'an XII par les lois susmentionnées, les cimetières affectés aux sépultures des défunts de confession protestante existaient dans certaines communes. C'est ainsi qu'aux abords de la Faculté de droit de Caen se trouve un célèbre cimetière protestant abritant la dernière demeure du dandy Georges Brummell.

L'abrogation de la disposition décrétale n'a pas eu pour effet de conduire ces communes à détruire lesdits cimetières. Mais, il leur est interdit aujourd'hui, du fait de la promulgation des lois abrogatives, non seulement d'en autoriser la création de nouveaux, mais aussi d'agrandir ceux existants ${ }^{125}$.

Dans ces cimetières, la neutralité religieuse ne s'impose pas dans la mesure où, dans le cadre de leurs limites actuelles, ils peuvent être totalement consacrés à un culte particulier.

Par suite, les associations cultuelles les gérant peuvent refuser d'y inhumer une personne n'étant pas considérée du culte auquel le cimetière est consacré.

De même, et parce que le décret du 10 février 1806 a déclaré les articles 22 et 28 du décret du 23 prairial, an XII, non applicables aux sépultures des personnes de religion juive, ce texte a incidemment autorisé la création de cimetières juifs privés.

Il existe aujourd'hui en France de tels cimetières qui peuvent encore recevoir des inhumations. Ils sont gérés soit par des associations, soit par des communautés religieuses sous l'autorité des consistoires départementaux.

D'ailleurs, aucune autorisation préfectorale n'est nécessaire pour l'inhumation dans ces cimetières ${ }^{126}$.

Par suite, les dirigeants légaux de la communauté israélite peuvent dès lors subordonner l'inhumation à une condition d'appartenance religieuse $\mathrm{e}^{127}$.

La légalité de l'existence de tels cimetières a été reconnue par le Conseil d'État ${ }^{128}$.

Toutefois, depuis les lois précitées de 1881 et 1884, il est interdit, même dans le cadre de cimetières privés, de créer de nouveaux cimetières juifs ou de les transférer ${ }^{129}$.

\section{b. Les carrés confessionnels}

À l'instar des cimetières protestants et juifs, les carrés juifs sont aujourd'hui une survivance, dont l'origine de ceux qui existent encore est antérieure aux lois de 1881 et 1884. Ils sont donc soumis au même statut que celui exposé ci-dessus pour lesdits cimetières ${ }^{130}$.

C'est pourquoi le juge administratif annule le refus d'un maire d'accorder une concession funéraire dans le carré juif d'un cimetière municipal lorsque ce refus est motivé exclusivement par la circonstance que les autorités religieuses juives déniaient au défunt la confession israélite. Cette jurisprudence administrative ${ }^{131}$ vise à nier la spécificité de telles parties du cimetière, voire, à plus ou moins long terme, à les faire disparaître.

Toutefois, et là encore ce n'est pas l'un des moindres paradoxes, diverses circulaires ${ }^{132}$ et réponses ministérielles à des questions parlementaires ${ }^{133}$ admettent que les autorités administratives, spécialement les maires, puissent procéder au regroupement de fait des sépultures des défunts, ayant manifesté cette volonté de leur vivant, dans un carré affecté à une religion.

Ce regroupement, précisent ces textes, doit respecter la neutralité religieuse du cimetière tant en ce qui concerne l'aspect extérieur de ses parties publiques qu'eu égard à la possibilité reconnue aux familles non croyantes ou ayant une autre croyance de s'y faire inhumer sans être dérangée par cette pratique.

Ainsi ne doit-il pas être séparé concrètement du reste du cimetière.

C’est donc un carré confessionnel matériellement réel mais apparemment virtuel que le « droit des circulaires et des réponses ministérielles » consacre!

Cette position officielle, souvent affirmée, est quelque peu en contradiction avec la jurisprudence précitée de 1993 ; bien qu'elle soit, au demeurant, toujours présentée dans ces «sources juridiques» comme conforme au principe de neutralité des cimetières.

Elle a d'ailleurs été reconnue par le Rapport Stasi qui réclame sa meilleure application!

Certes, elle vise surtout à assurer une meilleure intégration dans les cimetières publics des personnes de confession musulmane.

D’ailleurs, les musulmans souhaitent une pérennisation de cette pratique et surtout sa légalisation ${ }^{134}$.

124. O. Guillaumont, «Du principe de neutralité des cimetières et de la pratique des carrés confessionnels », JCP A, n 50, $2004, \mathrm{n}^{\circ} 1799, \mathrm{p} .1585$.

125. CE 18 août 1944, Lagarrigue, Rec. 237.

126. Rép. min. $n^{\circ} 8815$, JOAN Q, 8 août 1994, p. 4047.

127. CE 13 mai 1964, D ${ }^{\text {lle }}$ Eberstarck, Rec. 288, AJDA, 1964, p. 485 (obs. A. de Laubadère).

128. CA Pau 17 février 1959, Ville de Bayonne c. Association cultuelle israélite, GP, 1959, 2, 106 ; Cass. civ. $1^{\mathrm{e}}$, 20 février 1961, Bull. civ. I. $n^{\circ}$ 111 ; CA Aix-en-Provence, $1^{\text {er }}$ février 1971, AJDA, 1972, II, p. 111 (note M. M.) ; Cass. civ. $1^{\text {re }}, 11$ avril 1973, Bull. civ. I. ${ }^{\circ} 141$.

129. CE 20 janvier 1984, Association cultuelle israélite de Marseille c. $M^{\text {me }}$ Rouquette et autres, req. $\mathrm{n}^{\circ} 19.631$.

130. CE Ass. 17 juin 1938, Dame veuve Derode, Rec. 549; CAA Nancy 26 juin 2003, M. et $M^{m e}$ Robert X..., req. ${ }^{\circ} 98$ NCo2573.

131. TA Grenoble, 5 juillet 1993, Époux Darmon, JCP, 1994. II. nº 22.198 (note P.-H. Prélot).

132. Circulaires du ministre de l'Intérieur $\mathrm{n}^{\circ}$ 75-603 du 28 novembre 1975 et $\mathrm{n}^{\circ}$ 91-30 du 14 février 1991, in G. d'Abbadie et C. Bouriot, Code pratique des opérations funéraires, $3^{\mathrm{e}}$ éd., Paris, Le Moniteur, 2004, p. 651-654.

133. Rép. min. $\mathrm{n}^{\circ} 27.526, J O A N$ Q, 18 juin 1990, p. 2928 ; rép. min., $\mathrm{n}^{\circ} 36.347$, JOAN Q, 25 mars 1991, p. 1233 ; rép. min. $\mathrm{n}^{\circ} 59.726$, JOAN Q, 19 octobre 1992 , p. 4821 ; rép. min. $\mathrm{n}^{\circ} 8.815$, JOAN Q, 8 août 1994, p. 4264 ; rép. min. $\mathrm{n}^{\circ} 37.252$, JOAN Q, 10 janvier 2000, p. 224

134. G. d'Abbadie et C. Bouriot, Code pratique des opérations funéraires, p. 656. 
Mais « $[\ldots]$ si on reconnaît aujourd'hui aux musulmans le droit d'avoir leurs propres cimetières en vertu du principe de la liberté de religion et de culte, on voit mal comment on pourrait leur refuser demain les autres revendications du moment qu'elles se rattachent aussi à la liberté de religion et de culte ${ }^{135}$.

"On le voit, les demandes communautaires viennent remettre en cause nos certitudes juridiques ${ }^{136}$.

Pourquoi pas dans l'absolu?

Sauf qu'il convient de remarquer pour mieux le regretter, que ce qui est autorisé, ou plus exactement implicitement admis ou toléré, pour certains, est fermement interdit pour d'autres.

«Sans doute, le culte de la minorité ne doit pas être outragé, mais le culte de la majorité n'est pas moins respectable ${ }^{137}$.

Cette contradiction est juridiquement regrettable mais elle illustre aussi, sur un plan sociologique cette fois-ci, que les personnes publiques ont de plus en plus de mal à concilier le respect de la neutralité religieuse avec les attentes religieuses de plus en plus fortes de certaines parties de la population française, particulièrement dans un domaine aussi sensible que celui touchant à la mort.

«Il semble dès lors impossible de concilier en droit le principe de neutralité du cimetière et la présence des carrés confessionnels (dont la nécessité est pourtant reconnue). Peut-être conviendrait-il, dans le cadre de la modernisation attendue du droit des cimetières, de donner une nouvelle définition de la neutralité de cet espace public, afin que l'existence des carrés confessionnels reçoive une consécration législative indispensable?

Malheureusement, cette réforme nécessaire constituant une importante exception au principe constitutionnel de laïcité de l'État risquerait de subir la censure du juge constitutionnel éventuellement saisi !» ${ }^{138}$.

Il convient d'ailleurs de remarquer que, malgré l'illégalité des carrés confessionnels, lorsque la commune a décidé d'un créer un dans son cimetière, un ossuaire spécial pour ce carré est en général aménagé. Les restes inhumés dans l'ossuaire doivent avoir été préalablement introduits dans une boîte à ossements ${ }^{139}$.

Ainsi, la gestion des lieux funéraires ébranle plus qu'elle ne remet véritablement en cause le principe de neutralité religieuse des lieux publics.

Il en va toutefois bien différemment pour le statut des lieux publics cultuels qui, du fait même de leur affectation, contestent ce principe.

\section{B. Un principe contesté : les lieux publics cultuels}

\section{Les aumôneries}

Selon l'article 2 de la loi du 9 décembre 1905, «Les dépenses relatives à des services d'aumôneries et destinées à assurer le libre exercice des cultes dans des établissements tels que lycées, collèges, écoles, hospices, asiles et prisons » peuvent être prises en charge par l'État, les départements et les communes.

Le financement des aumôneries n'était donc à l'origine qu'une faculté voire qu'une éventualité, et en aucun cas, une obligation juridique pour les personnes publiques.

Mais, le Conseil d'État a estimé que le recours à un ministre du culte pour permettre aux malades d'exercer leur religion dans un établissement, même public, mais hospitalier, alors que le croyant est hors d'état d'être sorti, est en quelque sorte pour ce dernier un droit.

Par suite, et toujours au nom de la liberté de religion, et incidemment d'exercice des cultes, la haute juridiction administrative a estimé que priver des personnes se trouvant dans un tel état d'immobilité, même dans le cadre d'un service public, et par là même dans un lieu public, en principe neutres tous deux, portait atteinte à la libre manifestation par les fidèles de leurs opinions, en l'occurrence religieuses ${ }^{140}$.

Ce que le décret $n^{0} 74-27$ du 14 janvier 1974 confirme à partir du moment où les visites du ministre du culte sont sollicitées; «mais les visites systématiques à l'initiative des ministres du culte sont interdites ${ }^{141}$. «Les ministres du culte [officiant dans les établissements publics hospitaliers] appartiennent aux quatre grandes religions : catholique, israélite, protestante et musulmane ${ }^{142}$.

En effet, si le service public, et par là même son lieu d'exécution, doivent être neutres, il n'est en aucune façon admissible qu'au nom d'une telle double neutralité, les usagers d'un service public se voient privés de l'exercice de leur religion.

Cela reviendrait à permettre aux personnes publiques de s'immiscer dans les consciences, et incidemment d'entraver l'exercice d'une religion alors même qu'elles sont censées au nom de la séparation, certes de ne pas promouvoir un culte religieux quelconque, mais de les respecter tous et permettre aux croyants de l'exercer.

Les aumôneries ont ce rôle. C'est d'ailleurs pourquoi la présence de symboles religieux y est tolérée ${ }^{143}$.

C’est pourquoi la même solution «libérale» a été adoptée par le Conseil d'État pour les lycéens, et plus pré-

135. S.A. Aldeeb Abu Sahlieh, Cimetière musulman en occident. Normes juives, chrétiennes et musulmanes, Paris, L'Harmattan, 2002 , p. 160.

136. G. d'Abbadie et C. Bouriot, Code pratique des opérations funéraires, p. 657.

137. L. Aucoc, Conclusions sur CE 8 février 1868, Jousseaume, Rec., p. 140.

138. D. Dutrieux et F. Leclercq, Opérations funéraires et laïcité, p. 44.

139. Rép. min. ${ }^{\circ} 33616$, JOAN Q, 8 novembre 1999, p. 6469.

140. CE 6 juin 1947, Union catholique des hommes du diocèse de Versailles, Rec. 250.

141. Un siècle de laïcité, EDCE, $\mathrm{n}^{\circ} 55,2004$, p. 315.

142. V. Sevaistre, «Les "agents publics" des cultes », CFP, n 233, 2004, Les Personnes publiques face aux religions, p. 12.

143. A. Le Goff, La Neutralité religieuse de l'État..., p. 349-350. 
cisément les seuls internes de lycées ${ }^{144}$. Cette solution, par extension compréhensive de la jurisprudence administrative, est réputée aujourd'hui bénéficier à tous les internes de l'Éducation nationale. Ce que confirme l'article R 1412 du Code de l'éducation ${ }^{145}$.

Le juge administratif l'a affirmé en son temps en annulant une circulaire ministérielle supprimant toutes les aumôneries qui n'existaient pas avant 1939 parce que cet acte viole l'obligation incombant à l'État d'en créer notamment au bénéfice des élèves internes; en effet, cette suppression avait pour effet de "priver [ces derniers] de certains établissements visés par ladite circulaire de la possibilité de continuer librement la pratique de leur culte et de recevoir l'enseignement religieux ${ }^{146}$.

Cette jurisprudence a été confirmée peu de temps plus tard. Ainsi, est énoncée comme une obligation juridique à la charge du ministère de l'Éducation nationale la création d'un service d'aumônerie dans tous les établissements scolaires où leur institution s'avère nécessaire, pour ne pas dire indispensable, au libre exercice de leur culte par les élèves ${ }^{147}$.

«Dans les internats, la création est [donc] de droit. Elle répond ici parfaitement à l'esprit de la jurisprudence la plus ancienne précitée: sans aumônerie, l'accès au culte n'est pas possible, encore que l'on puisse remarquer que les autorisations de sortie telles qu'elles sont organisées actuellement permettent sans aucun doute une pratique dans une paroisse ${ }^{148}$.

Mais, cette obligation ne s'impose pas, en principe, au niveau des écoles primaires, et ce, en vertu de l'article R 141-1 du Code de l'éducation ${ }^{149}$. Il est vrai qu'il est plus rare que des enfants du primaire soient internes, sauf dans des cas particuliers tels que les enfants de bateliers ou d'enfants faisant l'objet d'une mesure d'assistance éducative, par exemple. Il convient de préciser que les «classes de neige » ou « les classes vertes» fonctionnent, en principe, sous un régime d'internat.

L'article R 141-4 de ce Code permet même une généralisation du recours aux aumôneries, même en l'absence d'internat. En effet, selon cet article, «Les lycées, collèges, et généralement tous établissements publics d'enseignement du niveau du second degré ne recevant pas d'internes et non encore pourvus d'un service d'aumônerie peuvent en être dotés, à la demande de parents d'élèves. La décision est prise par le recteur dans des conditions et selon des procédures déterminées par arrêté du ministre chargé de l'éducation.

Si la sécurité ou la santé des élèves le justifie, le recteur peut, après avis du chef d'établissement, autoriser les aumôniers à donner l'enseignement religieux à l'intérieur des établissements".

Ce texte confirme la jurisprudence administrative $e^{150}$ selon laquelle le droit à la création d'une aumônerie au niveau de l'enseignement secondaire est une obligation juridique de l'État lorsque les enseignés, et surtout leurs parents, le réclament.

«La loi Debré du 31 décembre 1959, bien qu'elle soit selon son intitulé même, consacrée aux rapports entre l'État et les établissements d'enseignement privés, [n'a fait que conforter cette évolution du droit, puisqu'elle] comporte en son premier article un paragraphe aux termes duquel l'État "prend toutes dispositions utiles pour assurer aux élèves de l'enseignement public la liberté des cultes et de l'instruction religieuse" ${ }^{151}$.

Ce qui permet à la liberté religieuse de s'exprimer pour le moins convenablement dans l'enceinte de lieux publics.

Toutefois, n'y a-t-il pas une ambiguïté entre, d'une part, permettre de manière fort libérale, pour ne pas dire " laxiste ", la création d'aumôneries au sein des établissements publics scolaires, alors même que, dans de tels lieux publics, est prohibée désormais la présence de signes religieux $^{152}$ ?

Mais, «même lorsque les services d'aumônerie sont à l'intérieur des locaux scolaires, tout prosélytisme, distribution de tracts, conférence, permanence et affichage à caractère de propagande sont interdits ${ }^{153}$.

Ce qui rappelle que le lieu public tolère « en son sol» la présence de ministres du culte et l'expression de la liberté religieuse au seul bénéfice des usagers mais non pour permettre aux cultes d'investir la sphère publique.

L'équilibre à trouver doit être parfois subtil et la frontière sûrement difficile à déterminer au quotidien, d'autant plus qu'elle dépend essentiellement des acteurs en présence.

Le droit d'exercer sa religion, du fait d'empêchement propre à leurs conditions, est aussi reconnu aux militaires ${ }^{154}$

144. $\mathrm{CE}_{1}{ }^{\mathrm{er}}$ avril 1949, Comité catholique des parents d'élèves des lycées et collèges de Seine-et-Oise, Rec. 164.

145. «Dans les établissements publics d'enseignement comportant un internat, une aumônerie est instituée à la demande de parents d'élèves ».

146. CE Ass. $1^{\text {er }}$ avril 1949, Chaveneau, Rec. 161, D., 1949, p. 531 (concl. F. Gazier), S., 1949. III. 49 (note J. Delpech).

147. CE Sect. 28 janvier 1955, Association des aumôneries de l'enseignement public, Rec. 50.

148. C. Durand-Prinborgne, La Laïcité, p. 72.

149. «Dans les écoles élémentaires publiques, il n'est pas prévu d'aumônerie. L'instruction religieuse est donnée, si les parents le désirent, à l'extérieur des locaux et en dehors des heures de classe, dans le respect des dispositions des articles L. 141-3 et L. 141-4".

150. CE Sect. 28 janvier 1955, Association des aumôneries de l'enseignement public, préc. Voir aussi : CE Sect. 7 mars 1969, Ville de Lille, Rec. 141.

151. C. Durand-Prinborgne, La Laïcité, p. 71.

152. L'article $1^{\text {er }}$ de la loi ${ }^{\circ}$ 2004-228 du 15 mars 2004 encadrant, en application du principe de laïcité, le port de signes ou de tenues manifestant une appartenance religieuse dans les écoles, collèges et lycées publics a inséré, dans le Code de l'éducation, après l'article L. 141-5, un article L. 141-5-1 ainsi rédigé: «Dans les écoles, les collèges et les lycées publics, le port de signes ou tenues par lesquels les élèves manifestent ostensiblement une appartenance religieuse est interdit. Le règlement intérieur rappelle que la mise en œuvre d'une procédure disciplinaire est précédée d'un dialogue avec l'élève. »

153. V. Sevaistre, «Les "agents publics" des cultes», p. 13.

154. Aux termes des deux premiers alinéas de l’article 4 de la loi n 2005-270 du 24 mars 2005 portant statut général des militaires, pour les militaires, « Les opinions ou croyances, notamment philosophiques, religieuses ou politiques, sont libres. Elles ne peuvent cependant être exprimées qu'en dehors du 
et aux prisonniers ${ }^{155}$, bien que ces deux catégories d'agents publics, pour les premiers, et d'usagers, pour les seconds, de services publics (militaire pour le premier et pénitentiaire pour le second) évoluent dans des lieux publics.

Si la liberté religieuse des détenus est donc reconnue et peut s'exprimer dans les prisons, lieux publics fermés pour ses usagers, le juge administratif ne semble pas considérer comme possible en ces lieux publics particuliers la pratique religieuse en dehors de l'encadrement des autorités religieuses. C'est ainsi qu'il a annulé la sanction de 8 jours de cellule disciplinaire infligée à un détenu pour avoir participé à une prière collective non autorisée au motif que l'intéressé n'avait pas conscience de commettre une infraction ${ }^{156}$, tout en admettant implicitement la thèse de l'administration pénitentiaire, selon laquelle la pratique religieuse ne peut être ni libre, ni spontanée en prison, elle doit être autorisée et apparemment exclusivement encadrée par les aumôniers engagés à cet effet.

In fine, il importe de souligner l'ambiguïté du statut des aumôniers. En effet, si leur nomination relève principalement de la compétence des autorités publiques, ces dernières y procèdent en accord avec les autorités religieuses concernées. Dès lors, la nomination d'un aumônier est soumise à une double décision : celle de l'autorité religieuse et celle de l'autorité administrative civile ou militaire compétente. Par suite, tout retrait d'agrément à un aumônier par les autorités religieuses, dont il relève, constitue un motif certain de rupture de son contrat ${ }^{157}$. Voilà un cas finalement intéressant de collaboration entre les deux pouvoirs, temporel et spirituel, alors qu'au nom des termes de leur séparation, ils devraient s'ignorer. Mais, comment le pourraient-ils au vu des multiples questions communes à ces deux pouvoirs?

\section{Les édifices cultuels}

«La loi [du 9 décembre 1905] attribue aux personnes publiques (État, communes) la propriété de la plupart des édifices du culte achevés en 1905 [...], sans indemnisation $[\ldots] »{ }^{158}$. Par suite, «Les églises, temples et synagogues [acquis avant 1905], dont sont propriétaires les collectivités publiques font partie de leur domaine public ${ }^{159}$.

Les travaux qui y sont effectués sont des travaux publics ${ }^{160}$. Les personnes publiques propriétaires ne sont d'ailleurs pas tenues d'y procéder; mais si un dommage est causé par leur négligence, elles en sont responsables. Cette règle vaut tant pour les immeubles cultuels que pour les meubles ayant aussi une destination cultuelle ${ }^{161}$.

Il en va de même des édifices non cultuels (presbytères, évêchés ou séminaires), qui sont soumis à tous les impôts locaux, en vertu de l'article 24 de la loi du 9 décembre 1905, à la différence des lieux de culte qui, en application du même article, sont exonérés des impôts locaux fonciers.

Mais, les édifices cultuels réalisés après 1905 sont des propriétés exclusives, donc privées, des seules associations cultuelles ${ }^{162}$

Dès lors, les « lieux publics exclusivement cultuels » sont fort nombreux en France mais, du fait de leur destination, de leur "spécificité», ils ne sont nullement soumis à la neutralité religieuse et ne peuvent pas l'être au risque de nier leur raison d'être.

D'ailleurs, le lieu de culte est mis à la disposition du ministre du culte et des fidèles; ceux-ci bénéficient d'un droit réel sur ce lieu public cultuel. «Ce droit consiste en une jouissance de plein droit, gratuite, exclusive, perpétuelle, sauf désaffectation ${ }^{163}$.

"L'affectation légale au culte des édifices en cause apporte aux droits de la personne publique propriétaire des limitations très strictes. Elle se traduit par des interdits comme l'impossibilité, tant que l'édifice n’a pas été désaffecté, d'aliéner l'immeuble ou le mobilier le garnissant, de le démolir ou encore de modifier l'état des lieux, l'affectation pouvant impliquer l'intangibilité, mais aussi par des devoirs, tels l'obligation d'accepter les offres de concours des fidèles pour l'entretien de l'édifice ou d'employer, en cas d'incendie, la prime d'assurance au profit de la conservation de l'édifice ${ }^{164}$. Plus généralement, "l'église est juridiquement à la disposition exclusive [du

service et avec la réserve exigée par l'état militaire. Cette règle s'applique à tous les moyens d'expression. Elle ne fait pas obstacle au libre exercice des cultes dans les enceintes militaires et à bord des bâtiments de la flotte ». La fin du deuxième alinéa confirme donc le droit aux aumôneries pour les militaires, qui leur est reconnu depuis 1880. «Au sein de l'Armée, il existe des aumôniers à statut militaire et des aumôniers à statut civil, ainsi que des aumôniers civils bénévoles. [...] Trois cultes sont aujourd'hui concernés : les cultes catholique (254), israélite (49) et protestant (71)» (Un siècle de läcité, EDCE, $\mathrm{n}^{\circ} 55,2004$, p. 315). Là encore, le culte musulman pâtit plus qu'il ne bénéficie de la séparation des Églises et de l'État !

155. Quant aux prisonniers, c'est le Code de procédure pénale, en ses articles D 432 à D 439 qui leur reconnaît actuellement ce droit. En effet, et aux termes de l'article D 432 de ce Code, "Chaque détenu doit satisfaire aux exigences de sa vie religieuse, morale ou spirituelle. Il peut à ce titre participer aux offices ou réunions organisés par les personnes agréées à cet effet ». Et l'article D 433 ajoute que «le service religieux est assuré, pour les différents cultes, par des aumôniers désignés par le directeur régional qui consulte à cet effet l'autorité religieuse compétente, et après avis du préfet ». Ces aumôniers sont, cette fois-ci, de confession non seulement catholique (513), protestante (267) et israélite (64), mais ils sont aussi de culte musulman (69) et orthodoxes (3). Il y a aussi des bouddhistes (V. Sevaistre, «Les "agents publics" des cultes », p. 12).

156. TA Versailles 24 mars 2005, M. B.., AJDA, $\mathrm{n}^{\circ}$ 14, 2005, p. 751 (obs. M.-C. de Montecler).

157. CE Sect. 17 octobre 1980, Pont, Rec. 374, AJDA, 1981, p. 256 (concl. D. Labetoulle); CE Sect. 27 mai 1994, Bourges, Rec. 263, concl. M. Denis-Linton.

158. J.-M. Pontier, Droits fondamentaux et libertés fondamentales, p. 101.

159. CA Paris 16 mai 1933, D., 1934. II. p. 101 (note M. Waline).

160. CE 10 juin 1921, Commune de Monsegur, Rec. 573, S., 1921. III. 49 (concl. Corneille; note M. Hauriou), RDP, 1921, p. 361 (concl.; note G. Jèze), D., 1922. III. 26 (concl.)

161. CE 10 novembre 1911, Commune de Saint-Blancard, Rec. 1001, S., 1912. III. 1.

162. Un siècle de laïcité, EDCE, $\mathrm{n}^{\circ}$ 55, 2004, p. 301.

163. J.-M. Pontier, Droits fondamentaux et libertés fondamentales, p. 104.

164. Sinon, la commune bénéficierait, au détriment du culte concerné et de ses fidèles, d'un enrichissement sans cause, si elle encaissait l'indemnité d'assurance sans reconstruire l'édifice cultuel : CE 19 janvier 1914, Commune de Morée, S., 1921. III. 13. 
desservant], il a sur elle un droit personnel de jouissance ${ } 165{ }_{»} 166$.

En somme, «Si [le législateur de 1905] remettait aux communes la propriétés de ces édifices, il ne leur remettait pas une propriété complète, absolue, mais seulement [...] une propriété démembrée, grevée d'une servitude [...] d'affectation cultuelle. La commune est bien seule propriétaire de l'église, mais elle n'est pas seule à avoir des droits sur l'église ${ }^{167}$.

À partir du moment où un ministre du culte est une « autorité constituée par [sa] hiérarchie [religieuse], la direction du culte et la réglementation intérieure de l'édifice [cultuel lui appartiennent] ; et cette qualité d'organisateur du culte, il peut l'opposer, non seulement au public ayant accès dans [le lieu cultuel], mais encore à l'autorité administrative investie cependant de la police sur les églises $[\ldots]{ }^{168}$

En effet, le ministre du culte n'exerce pas un véritable et exclusif pouvoir de police. Dès lors, «Il ne sera soumis aux règlements de police du maire, pourvu que ceux-ci ne portent pas atteinte au libre exercice du culte $[\ldots] »{ }^{169}$.

Par suite, l'autorité légitime de police, le plus souvent le maire, ne peut intervenir dans le lieu de culte que si l'ordre public y est menacé à l'intérieur ${ }^{170}$. L'article 25 de la loi du 9 décembre 1905 rappelle d'ailleurs que les réunions cultuelles «[...] restent placées sous la surveillance des autorités dans l'intérêt de l'ordre public». C'est ainsi que le maire peut refuser de mettre à disposition d'un groupe intégriste une église ${ }^{171}$.

Mais, le ministre du culte détient un pouvoir de « garde et de police de l'église en vue d'assurer aux fidèles l'exer- cice de leur religion ${ }^{172}$. À cet effet, « [...] il pourra en vertu de ce droit d'occupant, exiger une clef de l'église, décider de l'affectation de telle ou telle partie de l'édifice aux diverses cérémonies, se servir du mobilier qui le garnit et agir en justice pour écarter les intrus, soit au possessoire, soit au pétitoire ${ }^{173}$.

«Le Conseil d'État s'en tient à une maxime: au curé, tout l'ordre intérieur ${ }^{174}$.

Par suite, le ministre du culte :

- fixe les horaires d'ouverture et de fermeture ${ }^{175}$;

- détient les clés de l'édifice cultuel : ainsi, le maire n'a pas le pouvoir de faire ouvrir la porte de l'église par un serrurier afin d'y introduire un convoi funèbre et y présider une cérémonie dans le cadre d'un enterrement civil ${ }^{176}$. Toutefois, le ministre du culte ne peut pas s'opposer à ce que le maire exerce son droit de contrôle sur l'église afin de procéder aux réparations de l'édifice;

- décide de l'aménagement et de la décoration intérieurs ${ }^{177}$;

- règle les cérémonies et peut donc refuser d'y procéder : il est seul responsable du bon déroulement des cérémonies cultuelles ${ }^{178}$;

- et assure la discipline du culte à l'intérieur : c'est ainsi que, dans l'église, le ministre du culte a le droit d'assurer l'ordre et d'y maintenir la décence et la tranquillité, et ce, parce qu'il y est investi du pouvoir d'organiser les cérémonies cultuelles dans son église ${ }^{179}$.

Mais, pour exercer toutes ces compétences, encore faut-il que le ministre du culte soit investi de la confiance des autorités religieuses légitimes dont il dépend ${ }^{180}$.

165. Corneille, Conclusions sur CE 20 juin 1913, Abbé Arnoud, Rec. 716.

166. Un siècle de laïcité, EDCE, $\mathrm{n}^{\circ}$ 55, 2004, p. 303-304.

167. Corneille, Conclusions sur CE 26 juin 1914, Vital Pichon, S., 1921. III. 14.

168. R. Durnerin, De la situation juridique des édifices domaniaux affectés au culte catholique d'après la jurisprudence du Conseil d'État et de la Cour de cassation, Thèse, Paris, Jouve et $C^{\text {ie }}, 1923$, p. 61.

169. A. Mestre, Note sous Cass. civ. 5 février 1912, Colonna et Abbé Forcioli, S., 1912. I. 355.

170. Il peut ainsi intervenir, mais là encore seulement au titre de la sécurité publique, pour fermer le lieu cultuel, lorsque ce dernier présente de sérieux dangers d'effondrement (CE 26 mai 1911, Ferry, Rec. 698, S., 1913. III. 7; CE 28 novembre 1913, Abbé Gouhier, Rec. 1159). Il en irait de même, mais de façon cette fois-ci seulement provisoire, si des troubles s'étaient produits dans ce lieu (CE 22 novembre 1906, Abbé Voituret, S., 1907. III. 51 (note M. Hauriou)). Dans le même sens, « [...] la nécessité de la conservation de ce domaine justifie que la collectivité propriétaire réglemente l'accès aux parties d'un édifice cultuel où n'est célébrée aucune cérémonie religieuse " (J. Fialaire et É. Mondielli, Droits fondamentaux et libertés publiques, p. 473). «[...] il ne pourra donc user de ce pouvoir que dans des circonstances d'une gravité telle qu'on puisse dire que son intervention s'impose » (R. Durnerin, De la situation juridique..., p. 79). Si ce n'est pas le cas, le juge administratif annulera la décision de fermeture parce qu'elle aboutirait à priver les affectataires d'une façon définitive de leur lieu de culte et cela équivaudrait à une véritable désaffectation (CE 8 février 1908, Abbé Déliard, Rec. 128, S., 1908. III. 52 (concl. Chardenet)).

171. TA Nantes 2 juin 1977, AJDA, 1977, p. 632 (note F. Moderne).Voir aussi : CE 8 février 1908, Abbé Deliard, Rec. 128.

172. CE 24 mai 1938, Abbé Touron, Rec. 462.

173. A. Mestre, note sous Cass. civ. 5 février 1912, Colonna et Abbé Forcioli, S., 1912. I. 355.

174. G. Le Bras, «Le Conseil d’État régulateur de la vie paroissiale», p. 68. Voir aussi G.-D. Marillia, «Le pouvoir de police du curé», La Vie communale et départementale, $\mathrm{n}^{\circ} 803,1995$, p. 253-258 ( $1^{\text {re }}$ partie) et $\mathrm{n}^{\circ} 804,1995$, p. $283-287$ ( $2^{\mathrm{e}}$ partie).

175. CE 24 février 1912, Abbé Saralongue, Rec. 250 ; Tb. Correct. Montpellier 19 mars 1930, Abbé Mader, D., 1931. II. 133 (note M. Waline); CE Sect. 4 novembre 1994, Abbé Chalumey, JCP, 1995. II. nº 22.415.

176. CA Bourges 28 juin 1909, S., 1909. II. 241.

177. «[...] il peut décider de l'affectation de telle ou telle partie de l'église et assigner à certains fidèles des places réservées » (R. Durnerin, De la situation juridique..., p. 70). Voir aussi : CE Sect. 15 janvier 1937, de Bonnafous, Rec. 49: un maire ne peut pas s'opposer à un réaménagement du mobilier d'une église décidé par le curé.

178. TGI Argentan $1^{\text {er }}$ février 1973, Consorts Dauphin, JCP, 1973. II. $n^{\circ} 17.473$ (obs. H. Mazeaud).

179. Crim. $1^{\text {er }}$ décembre 1910, Abbé Galin, S., 1911. I. 346.

180. CE 8 avril 1911, Abbé Anselme, Rec. 461. Dans le même sens, voir : Cass. civ. 6 février 1912, Colonna et Abbé Forcioli, S., 1912. I. 352 (note A. Mestre), D., 1912. I. 121; CE 29 janvier 1920, Abbé Barraud, Rec. 75; CE 25 juin 1943, Église réformée de Marseille, D., 1944, p. 70 (note P. Reuter); CE 28 mars 1945 , Église réformée de Vigan, D., 1946, p. 156 (concl. Lagrange); CA Paris 13 juillet 1977, D., 1978, p. 458 (obs. Y. Geraldy); TA Nantes 2 juin 1977, AJDA, 1977. II. p. 632 (note F. Moderne) ; Cass. civ. 17 octobre 1978, Abbé Coache, Bull. civ. I. nº 308, D., 1979, p. 120 ; CE 24 avril 1981, Cousseran, RDP, 1982, p. 508 
Étant alors le représentant le plus autorisé du groupe des fidèles, c'est lui qui doit agir en justice afin de demander et d'obtenir, par voie de conséquence, l'expulsion d'un «ministre du culte intrus dans son lieu de culte ${ }^{181}$.

Par suite, le ministre du culte exerce ses compétences, et ceci, même contre les fidèles ${ }^{182}$. En effet, pour ces derniers, "À vrai dire, leurs droits se réduisent à peu de chose [...]. Mais, pour modestes qu'ils soient, ils n'en existent pas moins ${ }^{183}$. Ainsi ont-ils librement accès au lieu de culte et peuvent-ils y exercer leurs actes de piété et assister aux cérémonies religieuses, mais dans le respect de la discipline intérieure réglementée par le ministre du culte.

Quant aux sonneries de cloches - sujet encore aujourd'hui délicat -, en application de l'article 27 de la loi du 9 décembre 1905, le maire peut, en principe, les réglementer; mais le juge administratif veille à cette occasion à ce que la liberté religieuse ne s'en trouve pas entravée ${ }^{184}$.

Les autorités publiques peuvent, de plus, ordonner des sonneries de cloches en cas de péril (le tocsin en cas d'incendie ${ }^{185}$, par exemple), pour la fête nationale, ou encore, lors du passage dans une ville du président de la République. Mais, elles utilisent très exceptionnellement ce pouvoir; ce qui évite ainsi les interférences entre les pouvoirs civil et religieux pour le plus grand confort des deux!

D'ailleurs, en ordonnant à des membres du personnel communal de sonner les cloches, en dehors de cas de péril imminent ou d'usage local, mais notamment pour des enterrements civils, le maire commet une voie de fait ${ }^{186}$.

"Qu'il s'agisse de limitation des heures de sonneries ou de fixation de leur mode d'exécution, il [le juge administratif] n'hésite pas à annuler toutes dispositions qui lui paraissent, étant donné les circonstances de fait dans lesquelles elles ont été édictées, non justifiées par le souci d'assurer la sécurité ou la tranquillité publique et qui sont de nature à entraver ou à troubler le libre exercice du culte ${ }^{187}$.

« Le système actuel réalise donc un équilibre satisfaisant entre la liberté de célébration intérieure du culte et les exigences de la sécurité publique ${ }^{188}$.
Mais, il s'en évince avec évidence de l'exposé des pouvoirs du ministre du culte sur «son » édifice cultuel que les lieux publics cultuels échappent grandement pour ne pas dire complètement au principe de la neutralité religieuse, jusqu'à ne relever quant à leur organisation intérieure presque que du ministre du culte à la disposition duquel ce lieu est mis.

\section{Conclusion}

En matière de lieux publics, comme pour tout élément confronté à la liberté religieuse, «L'État est protecteur de l'exercice individuel et collectif de la liberté de conscience. La neutralité du service public est à cet égard un gage d'égalité et de respect de l'identité de chacun » ${ }^{189}$.

Mais, «Parce que l'intolérance et les préjugés se nourrissent de l'ignorance, la laïcité suppose également une meilleure connaissance réciproque y compris en matière de religion ${ }^{190}$.

«Peut être conviendrait-il de faire place dans les établissements publics scolaires à un enseignement de l'histoire des religions, ce qui ne serait pas contraire au principe général de laïcité ${ }^{191}$.

Ce qu'a failli reconnaître la loi $\mathrm{n}^{\circ}$ 2005-380 du 23 avril 2005 d'orientation et de programmation pour l'avenir de l'école si avait été maintenu dans son texte l'amendement présenté par le député-maire Jean-Pierre Brard, apparenté communiste; cet amendement a d'ailleurs été adopté presque à l'unanimité (moins une voix) à l'Assemblée nationale, le 18 février $2005^{192}$.

En somme, aujourd'hui, il y a un quasi-consensus sur la nécessité d'un «véritable» enseignement objectif et à dimension exclusivement culturelle du fait religieux ${ }^{193}$, sans, toutefois, que ne soient résolues les questions pourtant essentielles de " que doit contenir cet enseignement? ", «comment doit-il être dispensé?», et incidemment, "par qui ?».

Pourtant la voie de la connaissance passe par celle de la connaissance.

181. CE 28 juillet 1911, Abbé Rougegré et autres, Rec. 908, S., 1912. III. 97 (concl. Chardenet) ; Cass. civ. 6 février 1912, Colonna et Abbé Forcioli, S., 1912. I. 352 (note A. Mestre), D., 1912. I. 121.

182. CA Versailles 11 janvier 1988, D., 1988. IR. 34 .

183. R. Durnerin, De la situation juridique..., p. 59.

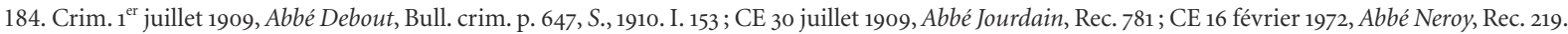

185. «Le recours à ce tocsin d'appel est désormais dépassé dans la plupart des communes : les sonneries sont remplacées par les appels des sirènes des centres de secours contre l'incendie, les mégaphones et les dispositifs divers de mise en alerte. Le recours aux sonneries de cloche est concevable dans les plus petites communes rurales» (C. Durand-Prinborgne, La Laïcité, p. 93).

186. TC 22 avril 1910 (Rec. 323) et 4 juin 1910 (Rec. 442), S., 1912. III. 129.

187. R. Durnerin, De la situation juridique..., p. 91-92.

188. G. Lebreton, Libertés publiques et droits de l'homme, p. 402.

189. Circulaire du 18 mai 2004 relative à la mise en ouvre de la loi no 2004-228 du 15 mars 2004 encadrant, en application du principe de laïcité, le port de signes ou de tenues manifestant une appartenance religieuse dans les écoles, collèges et lycées publics, JORF, 22 mai 2004 , p. 9033. 190. Ibid.

191. C. Leclercq, Libertés publiques, p. $322, \mathrm{n}^{\circ} 434$.

192. La Vie, 16 juin 2005, n 3120, p. 65.Voir aussi les débats sur le site www. assemblee-nationale. fr.

193. R. Debray, L'Enseignement du fait religieux à l'école, Paris, Odile Jacob, 2002.Voir aussi R. Mehdi, «L'Union européenne et le fait religieux», RFDC, $\mathrm{n}^{\circ} 54,2003$, p. $227-248$. 